\title{
Singularity development and supersymmetry in holography
}

\author{
Alex Buchel \\ Department of Applied Mathematics, University of Western Ontario, \\ London, Ontario N6A 5B\%, Canada \\ Department of Physics and Astronomy, University of Western Ontario, \\ London, Ontario N6A 5B\%, Canada \\ Perimeter Institute for Theoretical Physics, \\ Waterloo, Ontario N2J 2W9, Canada \\ E-mail: abuchel@uwo.ca
}

ABSTRACT: We study the effects of supersymmetry on singularity development scenario in holography presented in [1] (BBL). We argue that the singularity persists in a supersymmetric extension of the BBL model. The challenge remains to find a string theory embedding of the singularity mechanism.

KEYworDs: Black Holes in String Theory, Gauge-gravity correspondence, Spacetime Singularities

ARXIV EPRINT: 1705.08560 


\section{Contents}

1 Introduction 1

2 DG model in microcanonical ensemble $\quad 3$

2.1 Grand canonical ensemble (a review) 4

2.2 Microcanonical ensemble 6

2.3 Dynamics of DG-B model $\quad 7$

2.3.1 QNMs and linearized dynamics of DG-B model 10

$\begin{array}{lll}\text { 2.3.2 Fully nonlinear evolution of DG-B model } & 12\end{array}$

3 Supersymmetric extension of BBL model $\quad 13$

$\begin{array}{lll}3.1 & \text { Phase diagram and QNMs } & 14\end{array}$

$\begin{array}{ll}3.2 & \text { Dynamics of sBBL horizons } \\ \end{array}$

3.2.1 Dynamics of symmetric sBBL sector and its linearized symmetry breaking fluctuations 18

$\begin{array}{lll}3.2 .2 & \text { Unstable sBBL dynamics } & 20\end{array}$

4 Conclusions $\quad 22$

A Numerical setup $\quad 23$

A.1 DG-B model 23

A.2 sBBL model 24

\section{Introduction}

Horizons are ubiquitous in holographic gauge theory/string theory correspondence $[2,3]$. Static horizons are dual to thermal states of the boundary gauge theory [4], while their long-wavelength near-equilibrium dynamics encode the effective boundary hydrodynamics of the theory [5]. Typically, dissipative effects in the hydrodynamics (due to shear and bulk viscosities) lead to an equilibration of a gauge theory state - a slightly perturbed horizon in a dual gravitational description settles to an equilibrium configuration. If the initial perturbation away from thermal equilibrium is sufficiently strong, non-hydrodynamic modes participate in the equilibration process ${ }^{1}$ - perturbed bulk horizon relaxes via quasinormal modes [8-12]. We call horizons that relax via hydrodynamic modes that attenuate in space-time domain or via positively gapped quasinormal modes ${ }^{2}$ stable horizons. Bulk holographic dual with (dynamically) stable horizons describe boundary gauge theory states which are stable with respect to sufficiently small fluctuations.

\footnotetext{
${ }^{1}$ Even when not excited, non-hydrodynamic modes are important as they determine the convergence properties of the all-order effective hydrodynamic description $[6,7]$.

${ }^{2}$ I.e., the quasinormal modes with $\operatorname{Im}(\omega)<0$.
} 
Translationary invariant horizon can suffer an instability when a hydrodynamic mode in a system becomes unstable. An example of such an instability is discussed in [13]. In this case, the expected end-point of the evolution is a new inhomogeneous phase of the system. ${ }^{3}$ Alternatively, a horizon can be de-stabilized when a positively gapped nonhydrodynamic mode becomes unstable when one lowers energy (or temperature) $[15,16]$. This instability realizes a holographic dual of a spontaneous symmetry breaking in a meanfield approximation - the system undergoes a second-order phase transition towards a new stable phase with a finite density condensate of the originally unstable mode.

Above classification of (un)stable horizons in a holographic framework matches both the gravitational and the field theory intuition. However, there is more to the story. In [17, 18] a new instability of the translationary invariant holographic horizons was identified:

- while there is a linearized instability below some critical energy density (or temperature), triggered by a non-hydrodynamic mode spontaneously breaking a discrete symmetry,

- there is no candidate equilibrium state with a condensate of this unstable mode.

The dynamics of the model $[17,18]$ was extensively studied in [1] (BBL): both the presence of the linearized instability and the absence of the suitable equilibrium state for the evolution below the criticality was confirmed dynamically. Moreover, it was argued that the gravitational system evolves to a region of arbitrary large curvatures in the vicinity of the horizon, asymptotically turning such region singular in a finite time with respect to the boundary theory. The area density of the apparent horizon, associated with the non-equilibrium entropy density of the boundary gauge theory [19, 20] also diverges within a finite boundary time. This latter observation is significant as it precludes evolution of instabilities towards any finite entropy density spatially inhomogeneous equilibrium states as well - in other words, despite the fact that BBL dynamics occurs in a constrained phase space (spatial homogeneity and isotropy), the conclusion that the system evolves to a singularity, violating the weak cosmic censorship conjecture, is robust.

The BBL model is not a top-down holographic construction. Thus, one might wonder whether the phenomenon discovered in $[1,17,18]$ occurs in real string theory holographic examples. A particular aspect of the model is the unboundedness of the bulk scalar field potential. It was argued in [1] that there is no weak cosmic censorship conjecture violation once the potential is bounded. On the other hand, unbounded scalar potentials do occur in supersymmetric top-down holographic models (as e.g., in [21]). Additionally, the exotic critical phenomena of [17] was identified in supergravity model [21] (DG) in grand canonical ensemble. In this paper we partly address above questions.

Unfortunately, construction of a top-down holographic model realizing the dynamics of BBL model remains open: while the DG model is "exotic" in grand canonical ensemble, we study in section 2 the equilibrium properties and dynamics of DG model in microcanonical ensemble and show that it realizes a standard spontaneous symmetry breaking instability as in $[15,16]$. In section 3 we present a supersymmetric extension of the BBL model, which

\footnotetext{
${ }^{3}$ See $[14]$ for a recent discussion.
} 
exhibits the exotic phenomenon of $[17,18]$. This sBBL model does not have an equilibrium state below the criticality, and, as in BBL model, its homogeneous and isotropic states evolve towards asymptotically divergent expectation value of the symmetry breaking operator. The similarities and differences of the BBL and sBBL model are further highlighted in section 4. Some technical details are delegated to appendix A.

\section{DG model in microcanonical ensemble}

In [21] the authors studied equilibrium states of $d=3 \mathcal{N}=8$ superconformal gauge theory dual to $A d S_{4} \times S^{7}$ at a finite temperature and a finite chemical potential with respect to a diagonal $\mathrm{U}(1)_{R} \subset \mathrm{SO}(8)$ global symmetry within different consistent truncations of $D=11$ supergravity on $S^{7}$, allowing for different patterns of spontaneous global symmetry breaking. We consider the following two consistent truncations, with the effective actions

$$
S_{\mathrm{DG}-\mathrm{A} / \mathrm{B}}=\frac{1}{\kappa^{2}} \int_{\mathcal{M}_{4}} d x^{4} \sqrt{-\gamma} \mathcal{L}_{\mathrm{DG}-\mathrm{A} / \mathrm{B}}
$$

where $\kappa$ is a four dimensional gravitational constant related to the central charge $c$ of the boundary gauge theory at the ultraviolet fixed point as

$$
c=\frac{192}{\kappa^{2}}
$$

and

- "DG-A" model Lagrangian (2+2 equal charged scalars model in [21]) is:

$$
\begin{aligned}
\mathcal{L}_{\mathrm{DG}-\mathrm{A}}= & \frac{1}{2} R-\frac{1}{4}(\partial \sigma)^{2}-\frac{1}{2}\left(\partial \gamma_{1}\right)^{2}-\frac{1}{2}\left(\partial \gamma_{2}\right)^{2}-\frac{1}{2} \sinh ^{2} \gamma_{1}\left(A^{1}+\bar{A}^{0}\right)^{2} \\
& -\frac{1}{2} \sinh ^{2} \gamma_{2}\left(A^{1}-\bar{A}^{0}\right)^{2}-\frac{1}{4} \cosh (\sigma)\left[\left(\bar{F}^{0}\right)_{\mu \nu}\left(\bar{F}^{0}\right)^{\mu \nu}+\left(F^{1}\right)_{\mu \nu}\left(F^{1}\right)^{\mu \nu}\right] \\
& -\frac{1}{2} \sinh (\sigma)\left[\left(F^{1}\right)_{\mu \nu}\left(\bar{F}^{0}\right)^{\mu \nu}\right]-V
\end{aligned}
$$

with

$$
V=-2\left(2 \cosh \gamma_{1} \cosh \gamma_{2}+\cosh \sigma\right)
$$

- "DG-B" model Lagrangian (4 equal charged scalars model in [21]) is:

$$
\mathcal{L}_{\mathrm{DG}-\mathrm{B}}=\frac{1}{2} R-(\partial \phi)^{2}-\sinh ^{2} \phi\left(A^{1}\right)^{2}-\frac{1}{4}\left(F^{1}\right)_{\mu \nu}\left(F^{1}\right)^{\mu \nu}-V,
$$

with

$$
V=-2(2+\cosh (2 \phi)) \text {. }
$$

For the maximally supersymmetric quantization the dimensions $\Delta$ of the CFT operators $\mathcal{O}$ dual to bulk scalars $\left\{\gamma_{1}, \gamma_{2}, \sigma\right\}$ (DG-A model) are

$$
\Delta\left(\mathcal{O}_{\gamma_{1}}\right)=\Delta\left(\mathcal{O}_{\gamma_{2}}\right)=\Delta\left(\mathcal{O}_{\sigma}\right)=1
$$


and to the bulk scalar $\phi$ (DG-B model) is

$$
\Delta\left(\mathcal{O}_{\phi}\right)=1
$$

In both models $A^{1}$ is the bulk gauge field dual to $\mathrm{U}(1)_{R}$ global symmetry; the bulk scalars $\gamma_{i}$ and $\phi$ have the $\mathrm{U}(1)_{R}$ charge 1 , while $\sigma$ is $R$-symmetry neutral.

Note that DG-A model is invariant under the $\mathbb{Z}_{2}$ symmetry:

$$
\bar{A}^{0} \rightarrow-\bar{A}^{0}, \quad \sigma \rightarrow-\sigma, \quad \gamma_{1} \leftrightarrow \gamma_{2} .
$$

Its truncation to a $\mathbb{Z}_{2}$-even sector produces DG-B model with the identification

$$
\gamma_{1}=\gamma_{2} \equiv \phi
$$

\subsection{Grand canonical ensemble (a review)}

We focus on DG-A model; the construction for the DG-B model is a consistent truncation as explained in (2.10). Following [21], we set the chemical potential $\mu_{1}=1$ for $\mathrm{U}(1)_{R}$ symmetry, and for the other global U(1) (holographic dual to the bulk gauge field $\bar{A}^{0}$ ) $\bar{\mu}^{0}=0$, i.e., spatially homogeneous and isotropic thermal equilibrium states of the CFT are represented by a bulk geometry with the asymptotics:

$$
\begin{array}{ll}
A^{1}=a_{1}(r) d t, & a_{1}=\mu_{1}+\frac{q_{1}}{r}+\mathcal{O}\left(r^{-2}\right), \\
\bar{A}^{0}=\bar{a}_{0}(r) d t, & \bar{a}_{0}=\frac{\bar{q}_{0}}{r}+\mathcal{O}\left(r^{-2}\right),
\end{array}
$$

where $r$ is a standard asymptotic-AdS radial coordinate (see (2.14) below) and $\left\{q_{1}, \bar{q}_{0}\right\}$ determine the $\mathrm{U}(1)_{R} \times \mathrm{U}(1)$ charge densities, see (2.17). Imposing the supersymmetric quantization (2.7),

$$
\gamma_{i}=\frac{\gamma_{i(1)}}{r}+\mathcal{O}\left(r^{-3}\right), \quad \sigma=\frac{\sigma_{(1)}}{r}+\mathcal{O}\left(r^{-3}\right)
$$

we identify the expectation values of the dual operators

$$
\left\langle\mathcal{O}_{\gamma_{i}}\right\rangle=\gamma_{i(1)}, \quad\left\langle\mathcal{O}_{\sigma}\right\rangle=\sigma_{(1)}
$$

The background metric ansatz takes form

$$
d s_{4}^{2}=-2 r^{2} e^{-\beta(r)} g(r) d t^{2}+\frac{d r^{2}}{2 r^{2} g(r)}+r^{2}\left(d x_{1}^{2}+d x_{2}^{2}\right)
$$

with the following asymptotic expansions at infinity:

$$
\begin{aligned}
& g=1+\frac{1}{2 r^{2}}\left(\gamma_{1(1)}^{2}+\gamma_{1(2)}^{2}+\frac{1}{2} \sigma_{(1)}^{2}\right)+\frac{m}{r^{3}}+\mathcal{O}\left(r^{-4}\right), \\
& \beta=\frac{1}{2 r^{2}}\left(\gamma_{1(1)}^{2}+\gamma_{1(2)}^{2}+\frac{1}{2} \sigma_{(1)}^{2}\right)+\mathcal{O}\left(r^{-4}\right),
\end{aligned}
$$


where parameter $m$ is related to the energy density of the state, see (2.17). Assuming that a regular Schwarzschild horizon is located at $r=r_{h}$, we have asymptotic expansions in $\left(r-r_{h}\right)>0$ as

$$
\begin{aligned}
g & =g_{h}^{(1)}\left(r-r_{h}\right)+\cdots, & \beta & =\beta_{h}^{(0)}+\beta_{h}^{(1)}\left(r-r_{h}\right)+\cdots, \\
a_{1} & =a_{1, h}^{(1)}\left(r-r_{h}\right)+\cdots, & \bar{a}_{0} & =\bar{a}_{0, h}^{(1)}\left(r-r_{h}\right)+\cdots, \\
\gamma_{i} & =\gamma_{i, h}^{(0)}+\gamma_{i, h}^{(1)}\left(r-r_{h}\right)+\cdots, & \sigma & =\sigma_{h}^{(0)}+\sigma_{h}^{(1)}\left(r-r_{h}\right)+\cdots .
\end{aligned}
$$

Parameters in asymptotic expansions (2.11), (2.12), (2.15) and (2.16) determine the thermodynamic properties of an equilibrium $\mathrm{CFT}$ state, the energy density $\mathcal{E}$, the pressure $P=\frac{1}{2} \mathcal{E}$, the grand potential density $\Omega=-P$, the temperature $T$, the entropy density $s$, and the $U_{R}(1) \times \mathrm{U}(1)$ charge density $\left\{\rho_{1}, \bar{\rho}_{0}\right\}$ :

$$
\mathcal{E}=-2 m, \quad T=\frac{r_{h}^{2}}{2 \pi} g_{h}^{(1)} e^{-\beta_{h}^{(0)} / 2}, \quad s=2 \pi r_{h}^{2}, \quad \rho_{1}=-q_{1}, \quad \bar{\rho}_{0}=-\bar{q}_{0} .
$$

We use numerical shooting method developed in [22] with a radial coordinate

$$
x \equiv \frac{r_{h}}{r}, \quad x \in(0,1],
$$

to relate the boundary and the near-horizon asymptotics of the dual gravitational background. We reproduce the results reported in [21]:

- At any temperature $T>0$ there is a phase of the CFT with zero condensates ${ }^{4}(2.13)$ and a grand potential density $\Omega_{\mathrm{RN}}$ :

$$
T=\frac{12 r_{h}^{2}-\mu_{1}^{2}}{8 \pi r_{h}}, \quad \mathcal{E}=2 r_{h}^{3}\left(1+\frac{\mu_{1}^{2}}{4 r_{h}^{2}}\right)=-2 \Omega_{\mathrm{RN}}, \quad \rho_{1}=\mu_{1} r_{h},
$$

where $r_{h} \geq \mu_{1} / \sqrt{12}$.

- There is a critical temperature $T_{c}$ in the system separating phases with nonzero condensates $(2.13)$

$$
\frac{T_{c}}{\mu_{1}}=0.1739106(2),\left.\quad \frac{\rho_{1}}{\mu_{1}^{2}}\right|_{T=T_{c}}=0.5234403(5),\left.\quad \frac{\mathcal{E}}{\mu_{1}^{3}}\right|_{T=T_{c}}=0.5485552(5) .
$$

- A phase with

$$
\left\langle\mathcal{O}_{\gamma_{1}}\right\rangle=\left\langle\mathcal{O}_{\gamma_{2}}\right\rangle \equiv\left\langle\mathcal{O}_{\phi}\right\rangle, \quad\left\langle\mathcal{O}_{\sigma}\right\rangle=0, \quad \bar{\rho}_{0}=0,
$$

i.e., model DG-B, exists at $T \geq T_{c}$. As its grand potential density $\Omega_{\mathrm{DG}-\mathrm{B}}$ is

$$
\Omega_{\mathrm{DG}-\mathrm{B}} \geq\left.\Omega_{\mathrm{RN}}\right|_{\left\{T, \mu_{1}\right\}=\text { const }}
$$

DG-B model realizes the exotic thermodynamics discovered in [17] in grand canonical ensemble.

\footnotetext{
${ }^{4}$ This is just an electrically charged $A d S_{4}$ RN black brane.
} 

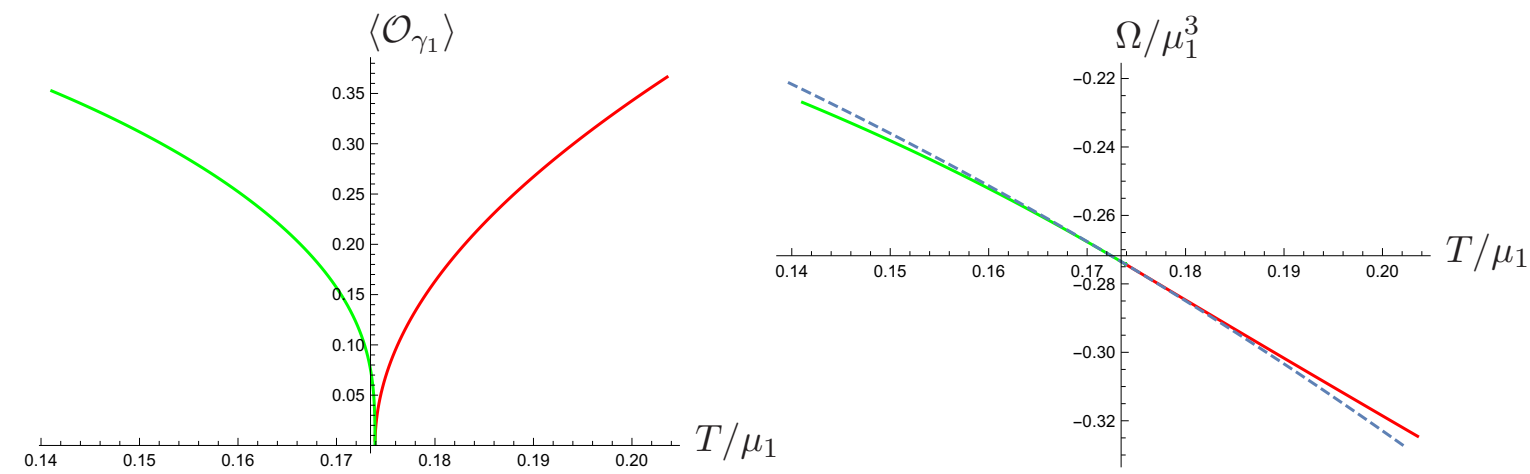

Figure 1. Left panel: expectation value $\left\langle\mathcal{O}_{\gamma_{1}}\right\rangle$ as a function of $T / \mu_{1}$ in model DG-A (green curve) and model DG-B (red curve). Right panel: grand potential densities $\Omega=\left\{\Omega_{\mathrm{DG}-\mathrm{A}}, \Omega_{\mathrm{DG}-\mathrm{B}}, \Omega_{\mathrm{RN}}\right\}=$ $\{$ (green curve), (red curve), (dashed blue curve) $\}$ as a function of $T / \mu_{1}$.

- A phase with

$$
\left\langle\mathcal{O}_{\gamma_{1}}\right\rangle \neq\left\langle\mathcal{O}_{\gamma_{2}}\right\rangle \neq 0, \quad\left\langle\mathcal{O}_{\sigma}\right\rangle \neq 0, \quad \bar{\rho}_{0} \neq 0,
$$

i.e., model DG-A, exists at $T \leq T_{c}$. As its grand potential density $\Omega_{\mathrm{DG}-\mathrm{A}}$ is

$$
\Omega_{\mathrm{DG}-\mathrm{A}} \leq\left.\Omega_{\mathrm{RN}}\right|_{\left\{T, \mu_{1}\right\}=\text { const }},
$$

DG-A model realized a standard holographic dual of a spontaneous symmetry breaking in grand canonical ensemble $[15,16]$.

$\left\langle\mathcal{O}_{\gamma_{1}}\right\rangle$ condensate in various phases of the CFT and the corresponding grand potential densities close to criticality, see (2.20), are presented in figure 1 .

\subsection{Microcanonical ensemble}

In previous section we reproduced some of the results of [21] to confirm that model DG-B indeed exhibits exotic thermodynamics in the spirit of [17] in grand canonical ensemble. The singularity mechanism identified in [1] hinges upon the persistency of exotic phase structure in microcanonical ensemble, i.e., in dynamical evolution with fixed energy density and charges. Unfortunately, the instability mechanism in both DG-A and DG-B models in microcanonical ensemble lead to standard picture of the spontaneous symmetry breaking $^{5}[15,16]$.

To study DG-A/B models in microcanonical ensemble, the only modification needed is the change of the boundary conditions on gauge fields in (2.11); now we require

$$
a_{1}=\mu_{1}+\frac{q_{1}}{r}+\mathcal{O}\left(r^{-2}\right), \quad \bar{a}_{0}=\bar{\mu}_{0}+\mathcal{O}\left(r^{-2}\right)
$$

where the "chemical potential" parameters $\left\{\mu_{1}, \bar{\mu}_{0}\right\}$ are adjusted to keep fixed $\mathrm{U}(1)_{R}$ charge density (parameter $q_{1}$ ), as well as the vanishing charge for the remaining $\mathrm{U}(1)$ (in model

\footnotetext{
${ }^{5}$ We verified that this remains true for supersymmetry breaking assignment of dimensions of bulk operators dual to $\left\{\gamma_{i}, \sigma\right\}$.
} 


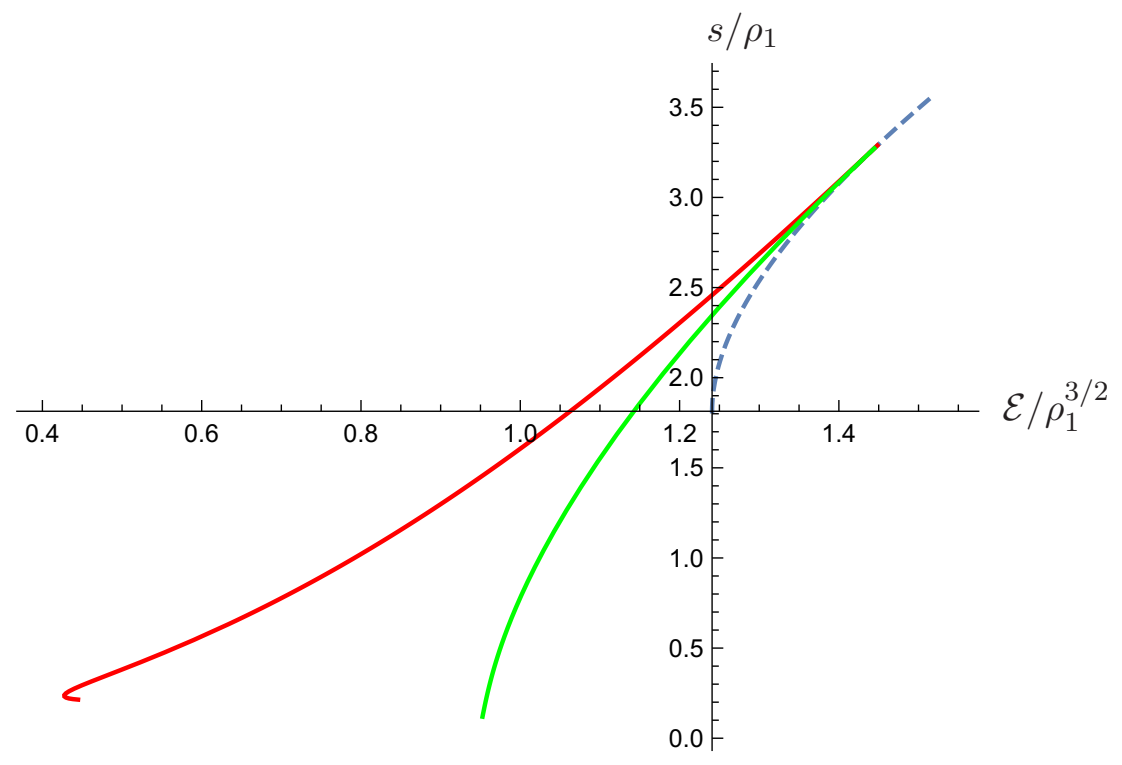

Figure 2. Entropy density $s$ as a function of the energy density $\mathcal{E}$ in model DG-A (green curve), DG-B (red curve) and the RN black brane (dashed blue curve).

DG-A). For numerical analysis we $\operatorname{set}^{6} \rho_{1}=-q_{1}=\frac{1}{2}-$ such a choice will put the phase transition numerically close to the one in grand canonical ensemble $(2.20)$ with $\mu_{1}=1$.

Phase diagram in microcanonical ensemble is presented in figure 2. The dashed blue curve represents symmetry unbroken phase - the RN black brane. The latter curve ends at the extremal RN solution:

$$
\left.\left\{\frac{\mathcal{E}}{\rho_{1}^{3 / 2}}, \frac{s}{\rho_{1}}\right\}\right|_{\mathrm{RN}, \text { extremal }}=\left\{\frac{2^{3 / 2}}{3^{3 / 4}}, \frac{2 \pi}{3^{1 / 4}}\right\} .
$$

In agreement with $(2.20)$, the symmetry unbroken phase becomes unstable at

$$
\left.\frac{\mathcal{E}}{\rho_{1}^{3 / 2}}\right|_{\text {crit }}=1.448503(6) \text {. }
$$

Two symmetry broken phases, i.e., model DG-A (green curve) and model DG-B (red curve) dominate entropically the symmetry unbroken phase for $\mathcal{E}<\mathcal{E}_{\text {crit }}$. Interestingly, it is the exotic phase of the grand canonical ensemble (model DG-B) that is realized in the microcanonical description of the CFT below the critical energy density.

\subsection{Dynamics of DG-B model}

In the previous section we argued that DG-B model describes the dominant phase of the CFT below the critical energy density in microcanonical ensemble. Here we describe dynamics of spatially homogeneous and isotropic states of the model. We confirm that the criticality in the model is a standard holographic realization of the spontaneous symmetry breaking.

\footnotetext{
${ }^{6}$ Because we are discussing states in the CFT, the precise choice is irrelevant in so far as we represent the data in dimensionless quantities.
} 
We begin with undoing the implicit bulk gauge symmetry fixing in the effective action of DG-B model (2.5):

$$
\mathcal{L}_{\mathrm{DG}-\mathrm{B}}=\frac{1}{2} R-(\partial \phi)^{2}-\sinh ^{2} \phi\left(\partial \theta-A^{1}\right)^{2}-\frac{1}{4}\left(F^{1}\right)_{\mu \nu}\left(F^{1}\right)^{\mu \nu}-V .
$$

We further introduce a pair of scalar fields $f_{i}$ in lieu of $\{\phi, \theta\}$ :

$$
f_{2}+i f_{1} \equiv e^{i \theta} \sinh \phi
$$

Assuming translational invariance along the spatial directions, we take

$$
\begin{aligned}
d s_{4}^{2} & =2 d t(d r-A(t, r) d t)+\Sigma(t, r)^{2}\left[d x_{1}^{2}+d x_{2}^{2}\right] \\
A^{1} & =a_{1}(t, r) d t, \quad f_{i}=f_{i}(t, r)
\end{aligned}
$$

leading for the following equations of motion

$$
\begin{aligned}
0= & \Sigma^{\prime \prime}+\frac{\Sigma}{1+f_{1}^{2}+f_{2}^{2}}\left(\left(f_{1} f_{2}^{\prime}-f_{2} f_{1}^{\prime}\right)^{2}+\left(f_{1}^{\prime}\right)^{2}+\left(f_{2}^{\prime}\right)^{2}\right) \\
0= & a_{1}^{\prime \prime}+2 a_{1}^{\prime}(\ln \Sigma)^{\prime}-2 f_{1}^{\prime} f_{2}+2 f_{1} f_{2}^{\prime}, \\
0= & d_{+}^{\prime} f_{1}+\frac{d_{+} f_{1}}{1+f_{1}^{2}+f_{2}^{2}}\left((\ln \Sigma)^{\prime}-f_{1}\left(f_{1}^{\prime}\left(1+f_{2}^{2}\right)-f_{2}^{\prime} f_{1} f_{2}\right)\right) \\
& +\frac{d_{+} f_{2} f_{1}}{1+f_{1}^{2}+f_{2}^{2}}\left(\left(f_{1}^{\prime} f_{1} f_{2}-f_{2}^{\prime}\left(1+f_{1}^{2}\right)\right)\right. \\
& +\frac{f_{1}^{\prime}}{\Sigma} d_{+} \Sigma+a_{1}\left(f_{2} f_{1} f_{1}^{\prime}-\left(1+f_{1}^{2}\right) f_{2}^{\prime}-f_{2}(\ln \Sigma)^{\prime}\right) \\
& -\frac{1}{2} f_{2} a_{1}^{\prime}+2 f_{1}\left(1+f_{1}^{2}+f_{2}^{2}\right), \\
0= & d_{+} f_{2}^{\prime}+\frac{d_{+} f_{2}}{1+f_{1}^{2}+f_{2}^{2}}\left((\ln \Sigma)^{\prime}+f_{2}\left(f_{1}^{\prime} f_{1} f_{2}-f_{2}^{\prime}\left(1+f_{1}^{2}\right)\right)\right) \\
& -\frac{d_{+} f_{1} f_{2}}{1+f_{1}^{2}+f_{2}^{2}}\left(f_{1}^{\prime}\left(1+f_{2}^{2}\right)-f_{2}^{\prime} f_{1} f_{2}\right) \\
& +\frac{f_{2}^{\prime}}{\Sigma} d_{+} \Sigma+a_{1}\left(f_{1}^{\prime}\left(1+f_{2}^{2}\right)-f_{2}^{\prime} f_{1} f_{2}+f_{1}(\ln \Sigma)^{\prime}\right) \\
& +\frac{1}{2} f_{1} a_{1}^{\prime}+2 f_{2}\left(1+f_{1}^{2}+f_{2}^{2}\right), \\
0= & A^{\prime \prime}-2 \frac{d_{+} \Sigma}{\Sigma^{2}} \Sigma^{\prime}-\left(a_{1}^{\prime}\right)^{2}+\frac{2 d_{+} f_{1}}{1+f_{1}^{2}+f_{2}^{2}}\left(f_{1}^{\prime}\left(1+f_{2}^{2}\right)-f_{2}^{\prime} f_{1} f_{2}\right) \\
& -\frac{2 d_{+} f_{2}}{1+f_{1}^{2}+f_{2}^{2}}\left(f_{1}^{\prime} f_{1} f_{2}-f_{2}^{\prime}\left(1+f_{1}^{2}\right)\right)-2 a_{1}\left(f_{1}^{\prime} f_{2}-f_{1} f_{2}^{\prime}\right),
\end{aligned}
$$

together with the constraint equations:

$$
\begin{aligned}
& 0=d_{+}^{\prime} \Sigma+d_{+} \Sigma(\ln \Sigma)^{\prime}-3 \Sigma+\Sigma\left(\frac{1}{4}\left(a_{1}^{\prime}\right)^{2}-2 f_{1}^{2}-2 f_{2}^{2}\right) \\
& 0=d_{+}^{2} \Sigma-2 A d_{+}^{\prime} \Sigma-\frac{d_{+} \Sigma}{\Sigma^{2}}\left(A \Sigma^{2}\right)^{\prime}+\frac{\Sigma}{1+f_{1}^{2}+f_{2}^{2}}\left(d_{+} f_{1}^{2}+d_{+} f_{2}^{2}+\left(f_{1} d_{+} f_{2}-f_{2} d_{+} f_{1}\right)^{2}\right)
\end{aligned}
$$




$$
\begin{aligned}
& -\frac{1}{2} A \Sigma\left(a_{1}^{\prime}\right)^{2}+2 a_{1}\left(d_{+} f_{2} f_{1}-f_{2} d_{+} f_{1}\right) \Sigma+\left(f_{1}^{2}+f_{2}^{2}\right) \Sigma a_{1}^{2}+2\left(2 f_{1}^{2}+2 f_{2}^{2}+3\right) A \Sigma, \\
0= & d_{+}^{\prime} a_{1}+\frac{2 a_{1}^{\prime}}{\Sigma} d_{+} \Sigma-2 d_{+} f_{2} f_{1}+2 f_{2} d_{+} f_{1}-A^{\prime} a_{1}^{\prime}-2 a_{1}\left(f_{1}^{2}+f_{2}^{2}\right),
\end{aligned}
$$

where $^{\prime} \equiv \partial_{r}$ and $d_{+} \equiv \partial_{t}+A \partial_{r}$. The constraint equations are preserved by the evolution equations provided they are satisfied at a given timelike surface - which in our case is the AdS boundary.

The general asymptotic boundary $(r \rightarrow \infty)$ solution of the equations of motion, given by $^{7}$

$$
\begin{aligned}
& \Sigma=r+\lambda(t)-\frac{f_{1,1}(t)^{2}+f_{2,1}(t)^{2}}{2 r}+\mathcal{O}\left(r^{-2}\right), \\
& A=(r+\lambda(t))^{2}-f_{1,1}(t)^{2}-f_{2,1}(t)^{2}-\dot{\lambda}(t)+\frac{A_{1}(t)}{r}+\mathcal{O}\left(r^{-2}\right), \\
& f_{1}=\frac{f_{1,1}(t)}{r}+\frac{f_{1,2}(t)}{r^{2}}+\mathcal{O}\left(r^{-3}\right), \\
& f_{2}=\frac{f_{2,1}(t)}{r}+\frac{f_{2,2}(t)}{r^{2}}+\mathcal{O}\left(r^{-3}\right), \\
& a_{1}=\frac{a_{1,1}(t)}{r}+\mathcal{O}\left(r^{-2}\right),
\end{aligned}
$$

is characterized by seven (generically time-dependent) parameters

$$
\left\{\lambda, f_{1,1}, f_{1,2}, f_{2,1}, f_{2,2}, A_{1}, a_{1,1}\right\} .
$$

The last two constraints in (2.32) imply that only five of them are independent:

$$
\begin{aligned}
0= & \dot{A}_{1}+2 \dot{\lambda}\left(f_{1,1}^{2}+f_{2,1}^{2}\right)+2 \lambda\left(f_{1,1} \dot{f}_{1,1}+f_{2,1} \dot{f}_{2,1}\right)-f_{2,1} \ddot{f}_{2,1}-f_{1,1} \ddot{f}_{1,1} \\
& +2 f_{2,1} \dot{f}_{2,2}+2 f_{1,1} \dot{f}_{1,2} \\
0= & \dot{a}_{1,1}-2 \dot{f}_{1,1} f_{2,1}+2 f_{1,1} \dot{f}_{2,1}-4 f_{1,1} f_{2,2}+4 f_{1,2} f_{2,1} .
\end{aligned}
$$

Restricting to static configurations, we identify $\left\{A_{1}, a_{1,1}\right\}$ with the energy density and the charge density as

$$
\mathcal{E}=-2 A_{1}, \quad \rho_{1}=-a_{1,1} .
$$

Dynamically, the constraint equations (2.35) become equivalent to energy and charge conservation, i.e.,

$$
\dot{\mathcal{E}}=0, \quad \dot{\rho}_{1}=0,
$$

provided $f_{i, 2}$ relate to $f_{i, 1}$ as follows:

$$
f_{i, 2}(t)=\frac{1}{2} \dot{f}_{i, 1}(t)-\lambda(t) f_{i, 1}(t) .
$$

As we will see, the boundary conditions (2.38) at late times, $t \rightarrow \infty$, correctly reproduce the equilibrium thermodynamics of DG-B model discussed in section 2.2. Following the

\footnotetext{
${ }^{7}$ Following [23] we used bulk gauge symmetry to require $a_{1} \sim \frac{1}{r}$ as $r \rightarrow \infty$.
} 
field redefinition (2.29) and relation to the expectation value of the dual operator (2.13), we identify

$$
\left\langle\mathcal{O}_{\phi}\right\rangle=\sqrt{f_{1,1}(t)^{2}+f_{2,1}(t)^{2}} .
$$

Additionally, $\mu_{1}$ in (2.25) is identified with

$$
\mu_{1}=\lim _{r \rightarrow \infty} \dot{\theta}=\frac{d}{d t}\left(\arctan \frac{f_{1,1}(t)}{f_{2,1}(t)}\right) .
$$

Finally, $\lambda(t)$ is the residual radial coordinate diffeomorphisms parameter

$$
r \rightarrow r+\lambda(t)
$$

which can adjusted to keep the apparent horizon at a fixed location, which in our case will be $r=1$ :

$$
\left.\left(\partial_{t}+A(t, r) \partial_{r}\right) \Sigma(t, r) \equiv d_{+} \Sigma(t, r)\right|_{r=1}=0 .
$$

To initialize evolution at $t=0$, we provide the bulk scalar profiles,

$$
f_{i}(t=0, r)=\mathcal{O}\left(\frac{1}{r}\right)
$$

along with the constant values of $\left\{A_{1}, a_{1,1}\right\}$, specifying the dual $C F T_{3}$ energy and charge densities according to (2.36). Eqs. (2.31) are employed to evolve such data (2.43) in time.

Further details of the numerical implementation can be found in appendix A.1.

\subsubsection{QNMs and linearized dynamics of DG-B model}

We discuss here the spectrum of QNMs associated with the spontaneous $\mathrm{U}(1)_{R}$ symmetry breaking and the linearized dynamics in DG-B model.

A symmetry unbroken phase of the boundary CFT is a $A d S_{4}$ RN black brane, which in notations (2.30), (A.1), (A.2) takes form:

$$
\sigma(t, x)=\lambda, \quad a(t, x)=\frac{x\left(4 m(\lambda x+1)+q_{1}^{2} x\right)}{4(\lambda x+1)^{2}},
$$

where $\lambda$ is determined from the stationarity of the horizon (2.42)

$$
0=4 \lambda^{2}\left(\lambda^{2}+4 \lambda+6\right)+(4 m+16) \lambda+q_{1}^{2}+4 m+4 .
$$

Introducing

$$
\begin{array}{rlrl}
f_{1}(t, x) & =-f(x) e^{\omega_{I} t} \sin \left(\omega_{R} t+g(x)\right), & f_{2}(t, x) & =f(x) e^{\omega_{I} t} \cos \left(\omega_{R} t+g(x)\right), \\
g^{\prime}(x) & \equiv d g(x), & (\ln f(x))^{\prime} \equiv \frac{1}{x}+l d f(x),
\end{array}
$$

where $\omega=\omega_{R}+i \omega_{I}$ is the symmetry breaking QNM frequency, we linearized the equations for the scalars $f_{i}$ on the gravitational background (2.44) to find

$$
\begin{aligned}
0= & l d f^{\prime}+l d f^{2}-d g^{2}+\left(4 \lambda^{2} x^{2}\left(\lambda^{2} x^{2}+4 \lambda x+6\right)+4 x\left(m x^{3}+4\right) \lambda+q_{1}^{2} x^{4}+4 m x^{3}+4\right)^{-1} \\
& \times\left(-4 \omega_{I}(\lambda x+1)(l d f \lambda x+l d f+\lambda)-4 d g(\lambda x+1)^{2} \omega_{R}-4 q_{1} x(\lambda x+1) d g\right.
\end{aligned}
$$




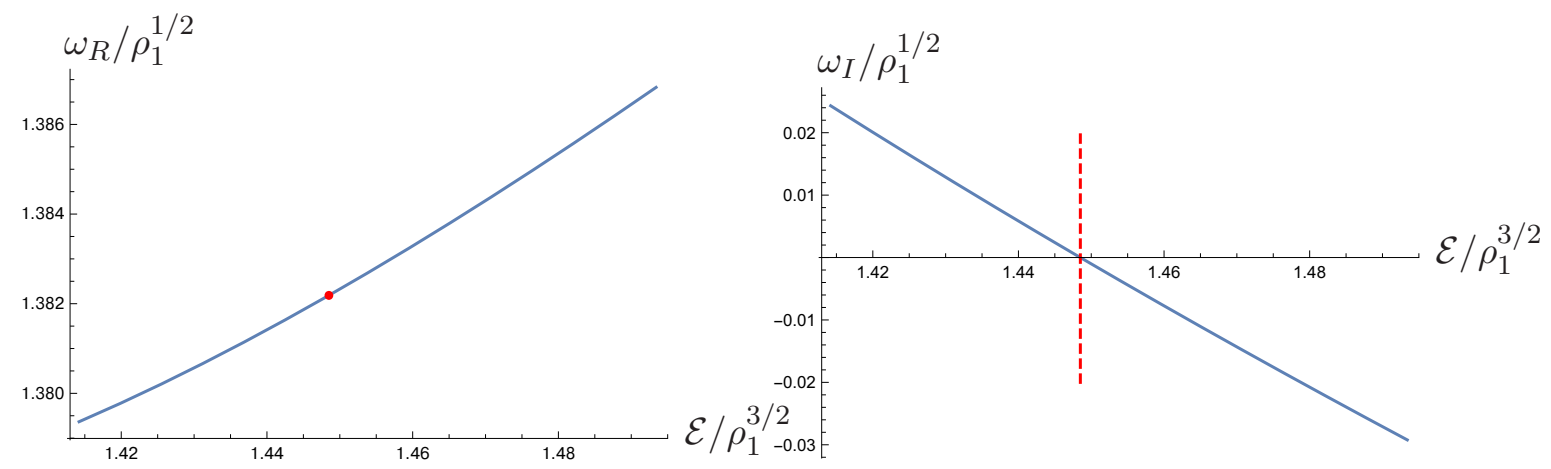

Figure 3. The spectrum of the $\mathrm{U}(1)_{R}$ symmetry breaking fluctuations at vanishing spatial momentum as a function of the energy density $\mathcal{E}$. The red dot (left panel) represents $\mu_{1}$ at critical energy density, see (2.20); the vertical red dashed line (right panel) represents the critical energy density, see (2.27).
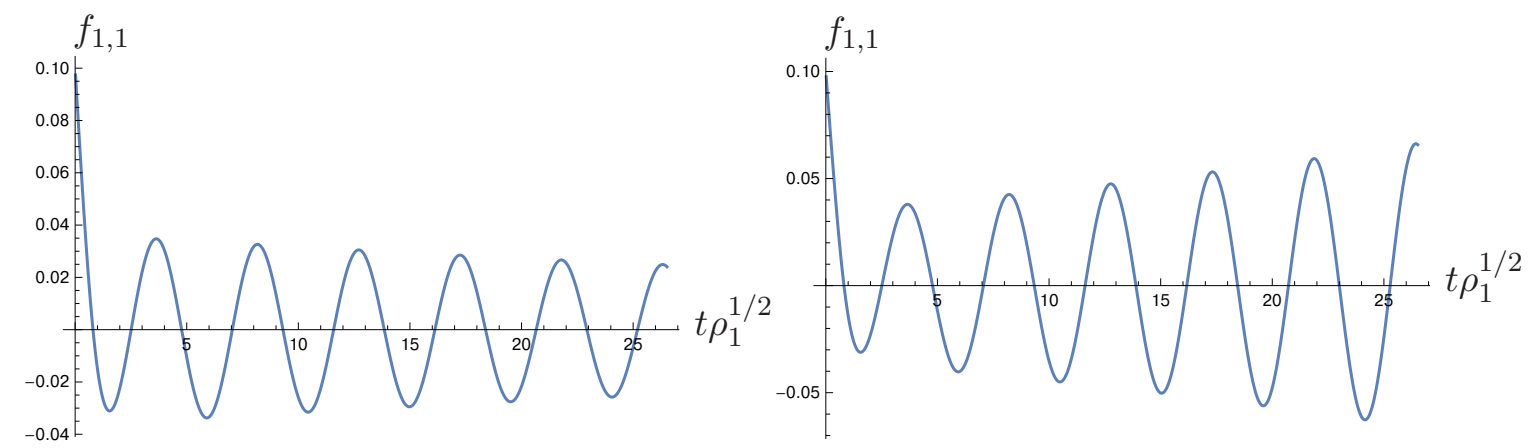

Figure 4. Linearized dynamics of the symmetry breaking scalars $f_{i}(t, x)$ on RN black brane background is captured by the evolution of the expectation value $f_{1,1}(t)$, see (2.33). Left panel corresponds to dynamics at energy density $\mathcal{E}=1.01538 \mathcal{E}_{\text {crit }}$ (symmetric phase is stable); right panel describes spontaneous symmetry breaking at $\mathcal{E}=0.976328 \mathcal{E}_{\text {crit }}$.

$$
\begin{aligned}
& +\left(16 \lambda^{2} x\left(\lambda^{2} x^{2}+3 \lambda x+3\right)+\left(16 m x^{3}+16\right) \lambda+4 x^{2}\left(q_{1}^{2} x+3 m\right)\right) l d f+8 \lambda^{2}(\lambda x+1)^{2} \\
& \left.+2 x\left(4 \lambda m x+q_{1}^{2} x+2 m\right)\right) \\
0= & d g^{\prime}+2 l d f d g+\left(4 \lambda^{2} x^{2}\left(\lambda^{2} x^{2}+4 \lambda x+6\right)+4 x\left(m x^{3}+4\right) \lambda+q_{1}^{2} x^{4}+4 m x^{3}+4\right)^{-1} \\
& \times\left(\left(4 \omega_{R}(\lambda x+1)\right)(l d f \lambda x+l d f+\lambda)-4 \omega_{I} d g(\lambda x+1)^{2}+\left(16 \lambda^{2} x\left(\lambda^{2} x^{2}+3 \lambda x+3\right)\right.\right. \\
& \left.\left.+\left(16 m x^{3}+16\right) \lambda+4 x^{2}\left(q_{1}^{2} x+3 m\right)\right) d g+4 q_{1} x(\lambda x+1) l d f+2 q_{1}(2 \lambda x+1)\right) .
\end{aligned}
$$

Eqs. (2.47) are solved subject to regularity at the location of the black brane horizon $(x=1)$, and the asymptotic $A d S_{4}$ boundary conditions $(x \rightarrow 0)$ following from $(2.38)$

$$
l d f=\frac{1}{2} \omega_{I}-\lambda+\mathcal{O}(x), \quad d g=-\frac{1}{2} \omega_{R}+\mathcal{O}(x) .
$$

The spectrum of the symmetry breaking QNM is presented in figure 3. Note that $\omega_{I}>0$, signalling the instability, once $\mathcal{E}<\mathcal{E}_{\text {crit }}$, see $(2.27)$.

As in [1], we verify our dynamical code for DG-B by "turning off" the scalar fields backreaction on the geometry. Scalar evolution in this case must reproduce at late times the quasinormal behaviour (2.46) with QNM spectrum presented in figure 3. Figures $4-5$ 

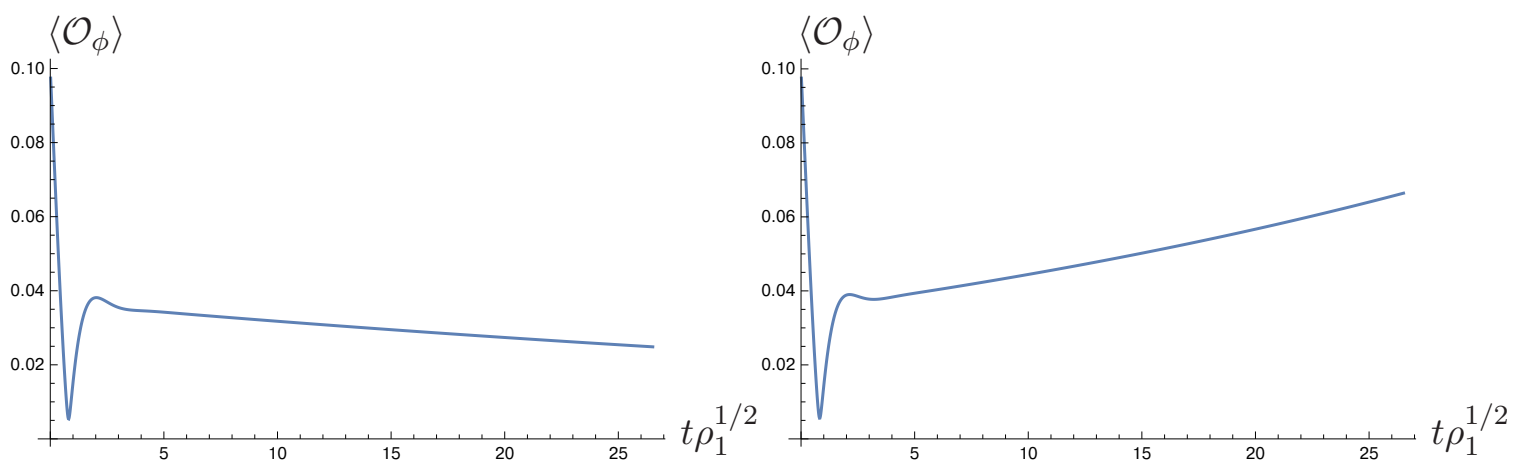

Figure 5. Same as in figure 4, except for the evolution of $\left\langle\mathcal{O}_{\phi}\right\rangle=\sqrt{f_{1,1}^{2}+f_{2,1}^{2}}$. The late time dynamics allows to extract $\omega_{I} \equiv \omega_{I, \text { fit }}$ to compare with the QNM behaviour, see (2.46).
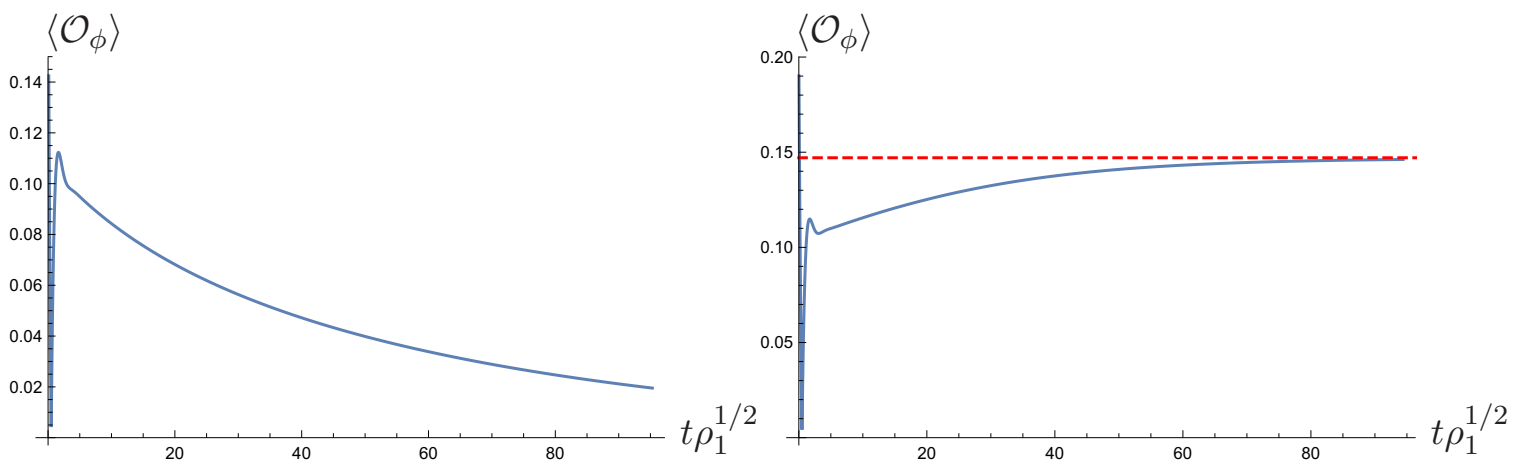

Figure 6. Fully nonlinear evolution of DG-B model. Left panel corresponds to dynamics at energy density $\mathcal{E}=1.01538 \mathcal{E}_{\text {crit }}$ (symmetric phase is stable); right panel describes spontaneous symmetry breaking at $\mathcal{E}=0.976328 \mathcal{E}_{\text {crit }}$. The red dashed line represents the energy density corresponding asymptotic (equilibrium) expectation value of the symmetry breaking operator $\mathcal{O}_{\phi}$ determined from microcanonical analysis of the model in section 2.2.

present the evolution of $f_{1,1}(t)$ and $\left\langle\mathcal{O}_{\phi}\right\rangle=\sqrt{f_{1,1}(t)^{2}+f_{2,1}(t)^{2}}$ for $\mathcal{E}>\mathcal{E}_{\text {crit }}$ (left panels stable case ) and $\mathcal{E}<\mathcal{E}_{\text {crit }}$ (right panels - unstable case). From (2.46) we expect

$$
\left\langle\mathcal{O}_{\phi}\right\rangle \propto e^{\omega_{I} t}
$$

Fitting the results in figure 5 at late times and comparing with the expected decay/growth from the earlier QNM computations at the corresponding energy densities we find

$$
\left|\frac{\omega_{I, \mathrm{fit}}}{\omega_{I, Q N M}}-1\right| \lesssim 10^{-7} .
$$

\subsubsection{Fully nonlinear evolution of DG-B model}

Microcanonical analysis of the DG-B model in section 2.2 and the linearized dynamics discussed in section 2.3.1 indicate that $\mathrm{U}(1)_{R}$ symmetry is spontaneously broken at $\mathcal{E}<\mathcal{E}_{\text {crit }}$ in the model, while at $\mathcal{E}>\mathcal{E}_{\text {crit }}$ the symmetric phase is the dominant one. As figure 6 presents, we find that this is indeed the case. Notice that approach to equilibrium is rather slow $\left(t_{\text {equilibration }} T \gtrsim 20\right.$ in this case $)$ - as emphasized in [12] this is expected in the sector of the gauge theory responsible for the critical behaviour close to transition. 
We confirm the conclusion reached in section 2.2: even though DG-B model exhibits an exotic thermodynamics discovered in [17] in grand canonical ensemble, it represents the well-known holographic realization of the spontaneous symmetry breaking $[15,16]$ in microcanonical ensemble.

\section{Supersymmetric extension of BBL model}

We would like to generalize BBL construction $[1,17,18]$ to a "supersymmetric" model. As we show, this is rather easy to achieve.

In four-dimensional gauged supergravity holographically dual to a supersymmetric boundary QFT $[24,25]$ the bosonic part of the effective action takes form

$$
S=\frac{1}{\kappa^{2}} \int_{\mathcal{M}^{4}} d x^{4} \sqrt{-\gamma}\left(\frac{1}{2} R+\mathcal{L}_{\text {bosonic }}\right)
$$

where the gravitational constant $\kappa$ relates to the central charge of the boundary theory as in $(2.2)$, and the Lagrangian $\mathcal{L}_{\text {bosonic }}$ of the scalar fields coupled to gravity is restricted to

$$
\mathcal{L}_{\text {bosonic }}=\mathcal{L}_{\text {kin }}-\mathcal{P}
$$

where the kinetic term is

$$
\mathcal{L}_{\text {kin }}=-K_{i j}\left\{\Phi_{k}\right\} \partial_{\mu} \Phi^{i} \partial^{\mu} \Phi^{j},
$$

and the potential is determined from the (real) superpotential $\mathcal{W}$

$$
\mathcal{P}=\left[K^{-1}\right]^{i j} \frac{\partial \mathcal{W}}{\partial \Phi^{i}} \frac{\partial \mathcal{W}}{\partial \Phi^{j}}-3 \mathcal{W}^{2} .
$$

Assuming the metric ansatz,

$$
d s_{4}^{2}=d r^{2}+e^{2 A(r)}\left(-d t^{2}+d x_{1}^{2}+d x_{2}^{2}\right)
$$

supersymmetric RG flow equations are obtained from the supersymmetry variation of a the fermions:

$$
\frac{d \Phi^{i}}{d r}= \pm\left[K^{-1}\right]^{i j} \frac{\partial \mathcal{W}}{\partial \Phi^{j}}, \quad \frac{d A}{d r}=\mp \mathcal{W} .
$$

Two comments are in order:

- the first order RG flow equations are consistent with the second order EOMs derived from the Lagrangian;

- because flow equations for scalars are of the first order, supersymmetry imposes specific quantization for the scaling dimensions for the dual operators (e.g., see (2.7) for DG-A model).

Recall, for the BBL model we have:

$$
\mathcal{L}_{\text {kin }}=-\frac{1}{4}(\partial \phi)^{2}-\frac{1}{4}(\partial \chi)^{2}, \quad \mathcal{P}_{\mathrm{BBL}}=-3-\frac{1}{2} \phi^{2}+\chi^{2}+\frac{1}{2} g \phi^{2} \chi^{2} .
$$


Note that the potential is unbounded from below for the nonlinear coupling $g<0$. The latter is necessary for the exotic phase structure of $[17,18]$. For a supersymmetric generalization of the model, we introduce the superpotential $\mathcal{W}$,

$$
\mathcal{W}=1+\frac{1}{4} \phi^{2}+\frac{1}{2} \chi^{2}+\frac{3}{160} \phi^{4}+\frac{3}{104} \chi^{4}+\left(\frac{1}{24}+\frac{g}{36}\right) \phi^{2} \chi^{2},
$$

leading to

$$
\mathcal{P}_{\mathrm{sBBL}}=\mathcal{P}_{\mathrm{BBL}}+\mathcal{O}\left(\phi^{n} \chi^{6-n}\right), \quad n \geq 0,
$$

i.e., the sBBL scalar potential captures the leading nonlinearity of the BBL model, but differs for higher order nonlinear interactions. As in BBL model, there is a single nonlinear coupling constant $g$. Explicitly,

$$
\begin{aligned}
\mathcal{P}_{\mathrm{SBBL}}= & \mathcal{P}_{\mathrm{BBL}}-\frac{1}{116812800}\left(520 g \chi^{2} \phi^{2}+540 \chi^{4}+780 \chi^{2} \phi^{2}+351 \phi^{4}\right)^{2} \\
& -\frac{9}{1600} \phi^{6}-\frac{45}{1352} \chi^{6}-\frac{1}{12960} \chi^{2} \phi^{4}\left(-160 g^{2}-372 g+531\right) \\
& -\frac{1}{16848} \phi^{2} \chi^{4}\left(-208 g^{2}-84 g+1071\right) .
\end{aligned}
$$

Notice that additional terms in $\mathcal{P}_{\mathrm{sBBL}}$ (compare to $\mathcal{P}_{\mathrm{BBL}}$ ) are trivially unbounded from below when

$$
-\frac{21+27 \sqrt{77}}{104}<g<0
$$

We explicitly verified that the second order equations of motion directly obtained from $\mathcal{L}_{\mathrm{SBBL}}$ are consistent with the corresponding supersymmetric RG flow equations (3.6). The supersymmetry imposes the following quantization on $\mathcal{O}_{r}$, dual to the bulk scalar $\phi$

$$
\Delta\left(\mathcal{O}_{r}\right)=1
$$

Notice that the quantization (3.12) is different from the one used in [1]; as a result, numerical implementation of sBBL model (see appendix A.2) is closer to that of DG-B model rather than the original $\mathrm{BBL}$ model.

\subsection{Phase diagram and QNMs}

Since BBL and sBBL models differ by higher-order nonlinearities in bulk scalar potentials, holographic renormalization for the models is the same. In particular, we can borrow the expressions for the thermodynamic quantities in [17], appropriate for the supersymmetric quantization (3.12). We present the results only — for detailed discussion follow $[1,17,18]$.

- Similar to [1], the holographic dual to gravitational sBBL model (3.1) is a massive QFT obtained as a deformation of the CFT (dual to $A d S_{4}$ ) with a central charge (2.2), by a relevant operator $\mathcal{O}_{r}$, dual to the bulk scalar $\phi$ (see (3.12)),

$$
\mathcal{H}_{\mathrm{CFT}} \longrightarrow \mathcal{H}_{\mathrm{CFT}}+\Lambda^{2} \mathcal{O}_{r},
$$

with $\Lambda$ being the deformation mass scale. $\Lambda \neq 0$ explicitly breaks the $\mathbb{Z}_{2}$ parity symmetry of the effective action (3.1): $\phi \leftrightarrow-\phi$. 


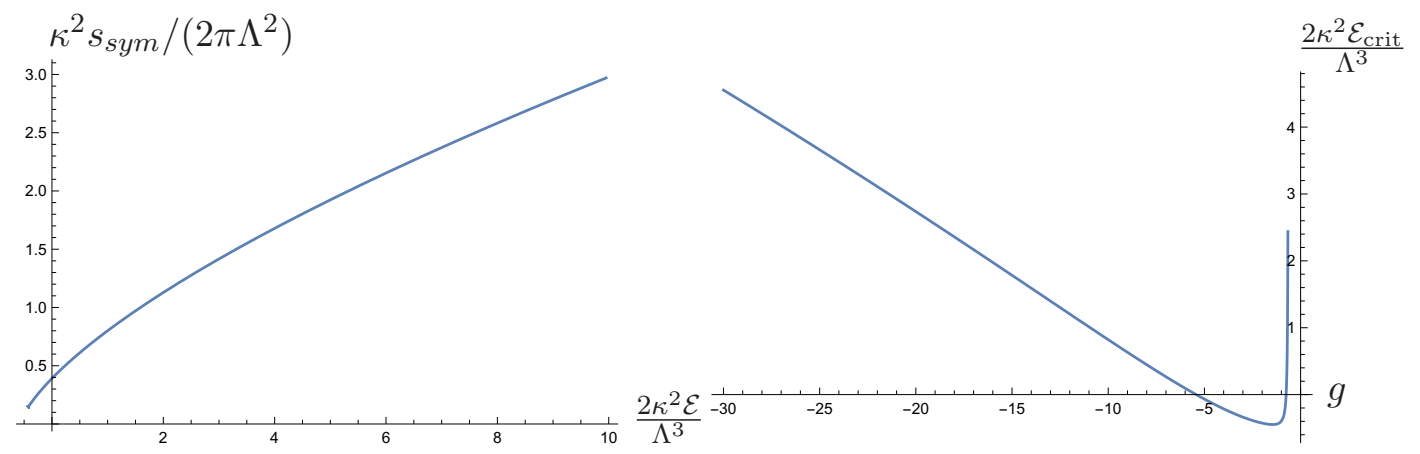

Figure 7. Entropy density $s_{\text {sym }}$ of the $\mathbb{Z}_{2}$-symmetric phase, i.e., with $\left\langle\mathcal{O}_{i}\right\rangle=0$, of sBBL model as a function of energy density $\mathcal{E}$ (left panel). As the energy density is decreased below the critical one $\mathcal{E}_{\text {crit }}$, the symmetric phase becomes perturbatively unstable with respect to linearized $\mathbb{Z}_{2}$-symmetry breaking fluctuations. The critical energy density for the onset of this instability as a function of the nonlinear coupling $g$ (see (3.10)) (right panel).
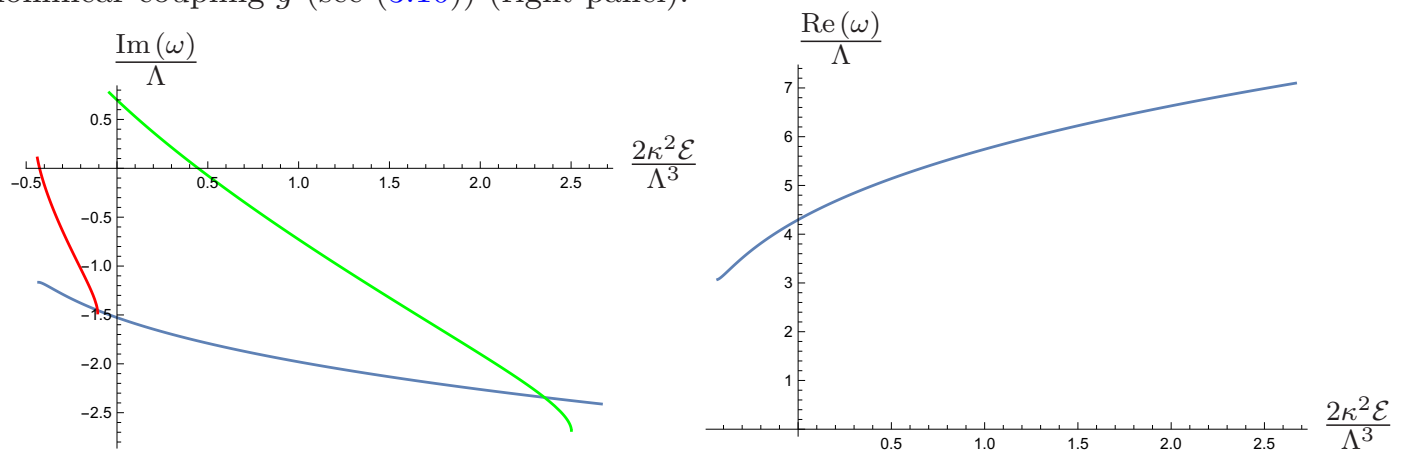

Figure 8. Left panel: spectrum of $\mathbb{Z}_{2}$ symmetry breaking QNM in sBBL model at $g=-2$ (red curve) and $g=-8$ (green curve) at vanishing spatial momentum. These QNMs have vanishing real part. The blue curves (both panels) represent the spectrum of QNM in sBBL model that connect to standard $\Delta=1 \mathrm{QNM}$ of $A d S_{4}$ black brane in the limit $\mathcal{E} / \Lambda^{3} \rightarrow \infty$.

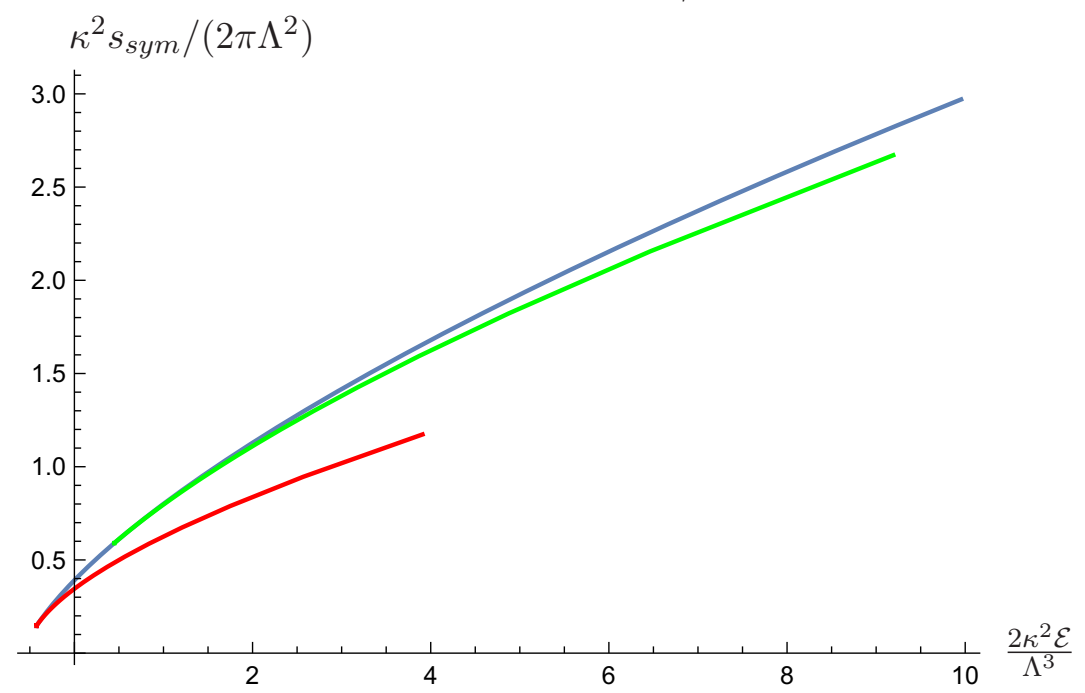

Figure 9. Phase diagram of sBBL model in microcanonical ensemble. The blue curve represents the symmetric phase of the system; the green and red curves are the exotic phases at $g=-8$ and $g=-2$ correspondingly, which exist only for $\mathcal{E}>\mathcal{E}_{\text {crit }}$. 
- There are two equilibrium phases of the sBBL model, distinguished by the symmetry property under $\chi \leftrightarrow-\chi$ : the symmetric phase with $\left\langle\mathcal{O}_{i}\right\rangle=0$, and the symmetry broken phase with $\left\langle\mathcal{O}_{i}\right\rangle \neq 0$.

- The entropy density of the symmetric phase $s_{s y m}$ as a function of the energy density $\mathcal{E}$ is presented in figure 7 (left panel). While this phase is thermodynamically stable $\frac{\partial^{2} \mathcal{E}}{\partial s_{s y m}^{2}}>0$, it is perturbatively unstable with respect to a linearized symmetry breaking fluctuations. The critical energy for the instability as a function of the coupling constant $g$ in (3.10) is shown in the right panel.

Notice that the energy density of the symmetric phase (as well as the critical energy density) can be negative. Typically, finite counterterms in holographic renormalization make the definition of the energy density ambiguous. This is the case for the $\phi$ scalar quantization with $\Delta\left(\mathcal{O}_{r}\right)=2$ : a finite boundary counterterm ${ }^{8}$

$$
\mathcal{L}_{\text {counter,finite }} \propto \int_{\partial \mathcal{M}_{4}} d x^{3} \sqrt{-h} \phi^{3},
$$

renders the definition of the energy density $\mathcal{E}$ ambiguous. However, for the supersymmetric quantization, i.e., (3.12), such term is not allowed, as it will violate the energy conservation Ward identity. So, the negative energy densities in the symmetric phase of the sBBL model are unambiguous. ${ }^{9}$ We discuss dynamics of sBBL model in section 3.2 for two representative values of the coupling constant $g=\{-2,-8\}$. Notice that $\mathcal{E}_{\text {crit }}(g=-2)<0$ and $\mathcal{E}_{\text {crit }}(g=-8)>0$. Additionally, the value $g=-2$ is within the range (3.11), where the gravitational scalar potential $\mathcal{P}_{\mathrm{sBBL}}$ is trivially unbounded from below.

- The spectrum of $\mathbb{Z}_{2}$ symmetry breaking quasinormal modes in sBBL model is presented in figure 8 as a function of energy density for $g=-2$ (red curve) and $g=-8$ (green curve) at vanishing spatial momentum. In agreement with results in figure 7 (right panel), these modes become unstable i.e., have a positive imaginary part, when $\mathcal{E}<\mathcal{E}_{\text {crit }}$ with

$$
\left.\frac{2 \kappa^{2} \mathcal{E}_{\text {crit }}}{\Lambda^{3}}\right|_{g=-2}=-0.42618(8),\left.\quad \frac{2 \kappa^{2} \mathcal{E}_{\text {crit }}}{\Lambda^{3}}\right|_{g=-8}=0.44726(8) .
$$

The unusual feature of these modes, first observed in [18], is the fact that they have vanishing real part, i.e.,

$$
\left.\operatorname{Re}(\omega)\right|_{\text {red \& green }}=0 .
$$

Because of (3.16), these modes must disappear from the spectrum in the limit $\frac{\mathcal{E}}{\Lambda^{3}} \rightarrow$ $\infty$, i.e., when sBBL approaches its UV (conformal) fixed point. ${ }^{10}$ The blue curves

\footnotetext{
${ }^{8} \sqrt{-h}$ is the induced metric on the cut-off surface $\partial \mathcal{M}_{4}$ in the holographic renormalization.

${ }^{9}$ It is easy to verify that for the supersymmetric RG flows (3.6) in sBBL model the (vacuum) energy density vanishes, as required by the boundary CFT supersymmetry.

${ }^{10}$ Recall that QNMs of a holographic dual to a CFT have both real and imaginary parts, typically with $\operatorname{Re}(\omega) \sim-\operatorname{Im}(\omega)[26]$.
} 
in figure 8 represent the sBBL QNM mode, which connects at asymptotically large energies to $\Delta=1 \mathrm{QNM}$ of the $C F T_{3}$. Notice that as $\mathcal{E}$ increases sufficiently far over the corresponding $\mathcal{E}_{\text {crit }}$, there is a "level crossing" (left panel), and the red and green curve QNMs cease to dominate the relaxation of the symmetry breaking fluctuations in the system - it is governed by the blue curve QNM.

- As in BBL model, in sBBL model new phases with spontaneous $\mathbb{Z}_{2}$ symmetry breaking appear in the microcanonical ensemble once $\mathcal{E}>\mathcal{E}_{\text {crit }}$, figure 9 . Once again, these new phases have lower entropy density than the symmetric phase (blue curve) and thus never dominate the dynamics of the system.

\subsection{Dynamics of sBBL horizons}

In section 3.1 we established that the equilibrium physics of BBL and sBBL models in microcanonical ensemble is identical: in both models the $\mathbb{Z}_{2}$ symmetric equilibrium phase becomes unstable below some critical energy density $\mathcal{E}_{\text {crit }}$; there is no end-point for the instability and the 'hairy' phases bifurcate from the onset of the instability towards $\mathcal{E}>$ $\mathcal{E}_{\text {crit }}$. These new phases are exotic - they have lower entropy density than the symmetry preserving phase and thus never dominate dynamically.

We now study the dynamics of the sBBL model. We highlight the main results and refer the reader to [1] for further implementation details. Because the quantization of the operator $\mathcal{O}_{r}$ dual to the bulk scalar $\phi$ is modified, see (3.12), the general asymptotic boundary $(r \rightarrow \infty)$ solution of the equations of motion is now given by

$$
\begin{aligned}
& \Sigma=r+\lambda(t)-\frac{1}{8} p_{1}(t)^{2} \frac{1}{r}+\mathcal{O}\left(\frac{1}{r^{2}}\right) \\
& A=\frac{r^{2}}{2}+\lambda(t) r-\frac{1}{8} p_{1}(t)^{2}+\frac{1}{2} \lambda(t)^{2}-\dot{\lambda}(t)+\frac{\mu}{r}+\mathcal{O}\left(\frac{1}{r^{2}}\right), \\
& \phi=\frac{p_{1}(t)}{r}+\left(p_{2}+\dot{p}_{1}(t)-\lambda(t) p_{1}(t)\right) \frac{1}{r^{2}}+\mathcal{O}\left(\frac{1}{r^{3}}\right), \\
& \chi=\frac{q_{4}(t)}{r^{4}}+\mathcal{O}\left(\frac{1}{r^{5}}\right) .
\end{aligned}
$$

It is characterized by two constants $\left\{p_{2}, \mu\right\}$, and three dynamical variables $\left\{p_{1}(t), q_{4}(t), \lambda(t)\right\}$. These parameters have the following interpretation:

- $p_{2}$ and $p_{1}(t)$ are identified with the deformation mass scale ${ }^{11} \Lambda$ and the expectation value of the relevant operator $\mathcal{O}_{r}$ of the dual $Q F T_{3}$,

$$
p_{2}=2^{-2 / 3} \Lambda^{2}, \quad p_{1}(t)=2^{-1 / 3}\left\langle\mathcal{O}_{r}(t)\right\rangle
$$

\footnotetext{
${ }^{11}$ The precise value of $\Lambda$ is not important - it sets the overall scale in the boundary QFT. Of course, care should be taken to keep $\Lambda$ time-independent. In what follows we measure all dimensionful quantities with respect to $\Lambda$. In numerical evolution we used $\Lambda \sim 1$, and adjusted its precise value for convenience. We verified that the results are independent of our $\Lambda$-choice.
} 

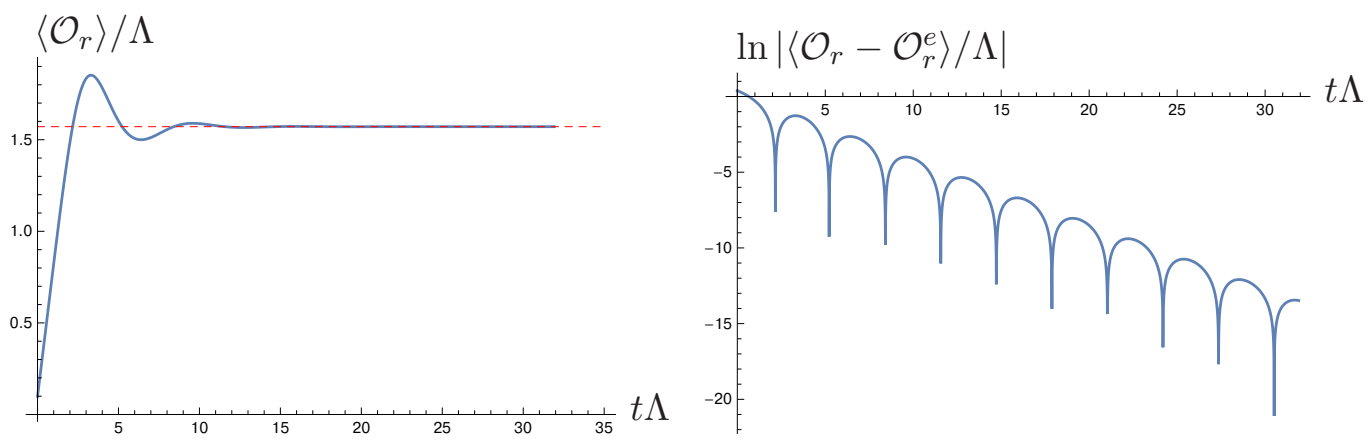

Figure 10. Typical relaxation in $\mathbb{Z}_{2}$ symmetric sector of sBBL model. The expectation value of $\mathcal{O}_{r}$ operator (dual to the bulk scalar $\phi$ ) settles to its equilibrium value $\mathcal{O}_{r}^{e}$ denoted by a dashed red line (left panel). Right panel shows a characteristic QNM ring-down of the expectation value to its equilibrium value.

- $q_{4}(t)$ is the normalizable coefficient of the bulk scalar $\chi$, identified with the expectation value of the $\mathbb{Z}_{2}$-symmetry breaking irrelevant operator $\mathcal{O}_{i}$ of the dual $Q F T_{3}$,

$$
q_{4}(t)=\left\langle\mathcal{O}_{i}(t)\right\rangle
$$

- $\mu$ is related to the conserved energy density $\mathcal{E}$ of the boundary $Q F T_{3}$ as follows

$$
\frac{2 \kappa^{2} \mathcal{E}}{\Lambda^{3}}=\frac{-4 \mu}{\Lambda^{3}}
$$

- $\lambda(t)$ is the residual radial coordinate diffeomorphisms parameter

$$
r \rightarrow r+\lambda(t)
$$

which can adjusted to keep the apparent horizon at a fixed location, which in our case will be $r=1$ :

$$
\left.\left(\partial_{t}+A(t, r) \partial_{r}\right) \Sigma(t, r) \equiv d_{+} \Sigma(t, r)\right|_{r=1}=0
$$

To initialize evolution at $t=0$, we provide the bulk scalar profiles,

$$
\phi(t=0, r)=\mathcal{O}\left(\frac{1}{r}\right), \quad \chi(t=0, r)=\mathcal{O}\left(\frac{1}{r^{4}}\right),
$$

along with the values of $\left\{p_{2}, \mu\right\}$, specifying the dual $Q F T_{3}$ mass scale $\Lambda$ (3.18) and the initial state energy density $\mathcal{E}(3.20)$.

Some details for the modification of the numerical code of [1] are collected in appendix A.2.

\subsubsection{Dynamics of symmetric sBBL sector and its linearized symmetry break- ing fluctuations}

Typical evolution in the symmetric sector (the bulk scalar $\chi$ identically vanishes) is shown in figure 10. An important check on the consistency of the evolution is the fact that the 

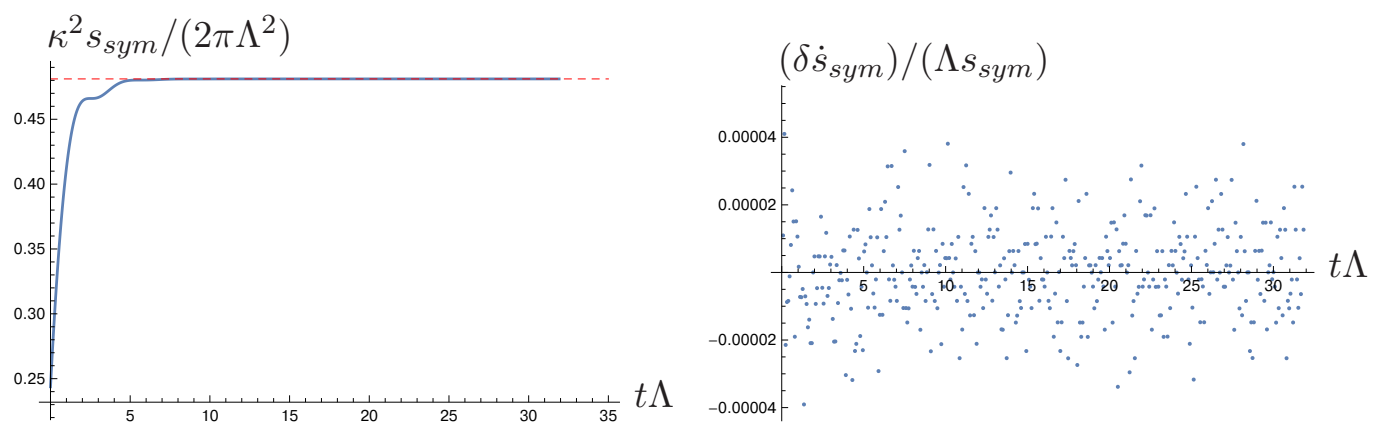

Figure 11. Evolution of the entropy density in $\mathbb{Z}_{2}$ symmetric sector of sBBL model. The red dashed line indicates the equilibrium value (left panel). The entropy production rate is always positive during the evolution, see (3.25). The right panel presents numerical error in computation of the entropy production rate, see (3.26).
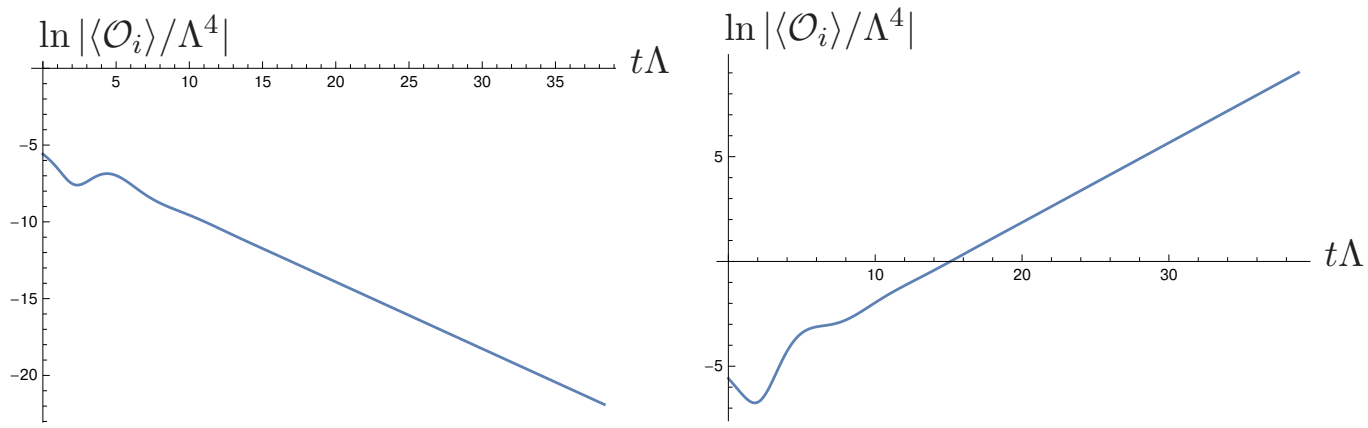

Figure 12. Linearized dynamics of the symmetry breaking expectation value $\left\langle\mathcal{O}_{i}\right\rangle$ for $\mathcal{E}<\mathcal{E}_{\text {crit }}$ (left panel) and $\mathcal{E}>\mathcal{E}_{\text {crit }}$ (right panel).

expectation value of $\mathcal{O}_{r}$ evolves at asymptotically late times to its equilibrium value at the corresponding energy density (here we take $g=-8$ and $\mathcal{E}=0.42978(1) \mathcal{E}_{\text {crit }}$ ), computed independently in section 3.1 .

One of the advantages of the holographic formulation of the dynamics of strongly interactive gauge theories is a natural definition of the non-equilibrium entropy density $s(t)$, associated with the area density of the apparent horizon,

$$
s(t)=\left.\frac{2 \pi}{\kappa^{2}} \Sigma(t, r)^{2}\right|_{r=1} .
$$

The evolution of the entropy density with time for the same set of parameters as in figure 10 is shown in figure 11. The red dashed line (left panel) indicates the equilibrium value of the entropy density at the corresponding energy density, computed in section 3.1. From the plot it is clear that the entropy production rate is non-negative, i.e., $\dot{s}_{s y m} \geq 0$. In fact, using the gravitational bulk equations of motion we find

$$
\dot{s}(t)=\left.\frac{\kappa^{2}}{2 \pi}\left(\Sigma^{2}\right)^{\prime} \frac{\left(d_{+} \phi\right)^{2}+\left(d_{+} \chi\right)^{2}}{-2 \mathcal{P}_{\mathrm{sBBL}}}\right|_{r=1},
$$

which can be analytically proven to be non-negative following [27]. Because a derivation of (3.25) involves gravitational bulk constraint equations not directly used in the numerical 

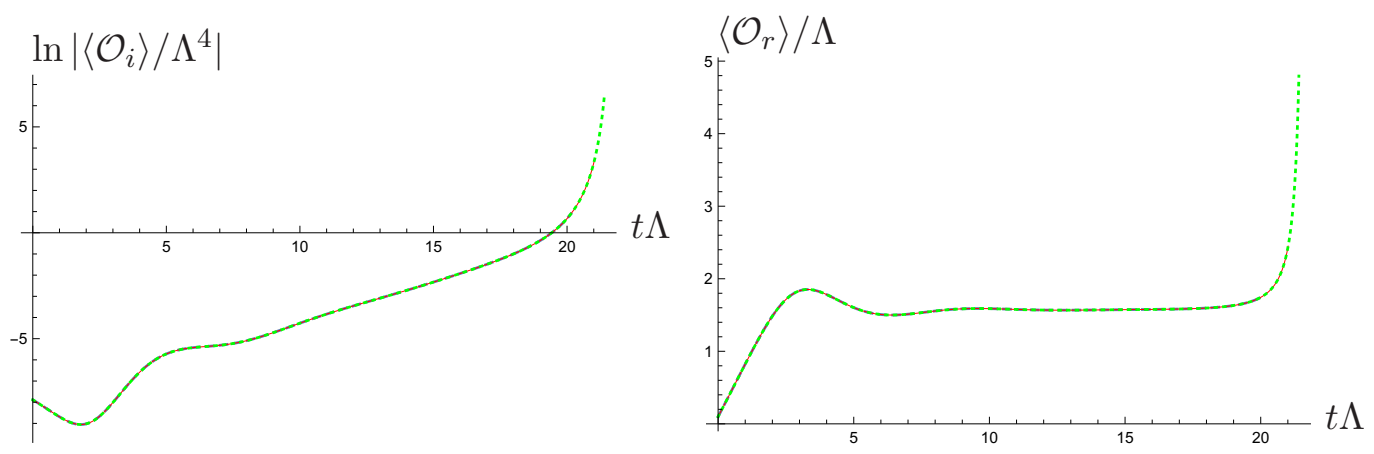

Figure 13. Evolution of expectation values of $\mathcal{O}_{i}$ and $\mathcal{O}_{r}$ operators in sBBL model with $g=-8$ in the unstable regime, i.e., with $\mathcal{E}<\mathcal{E}_{\text {crit }}$. Different color coding represents different spatial resolutions of the numerical runs.

evolution code, an important (dynamical) consistency check on the code is the vanishing of

$$
\left.\delta \dot{s} \equiv \frac{\kappa^{2}}{2 \pi}\left[\frac{d}{d t} \Sigma^{2}-\left(\Sigma^{2}\right)^{\prime} \frac{\left(d_{+} \phi\right)^{2}+\left(d_{+} \chi\right)^{2}}{-2 \mathcal{P}_{\mathrm{sBBL}}}\right]\right|_{r=1} .
$$

The right panel in figure 11 monitors the quantity (3.26).

Taking the value of the coupling $g=-8$ in the bulk scalar potential, we study next the linearized dynamics of the $\mathbb{Z}_{2}$ symmetry breaking fluctuations. Figure 12 presents the evolution of $\left\langle\mathcal{O}_{i}\right\rangle$ without the backreaction on the symmetric sector dynamics. Once the symmetric sector equilibrates, $t \Lambda \gtrsim 10$, the linearize $\mathbb{Z}_{2}$ breaking fluctuations decay (left panel: $\mathcal{E}=1.7191(2) \mathcal{E}_{\text {crit }}$ ) or grow (right panel: $\mathcal{E}=0.42978(1) \mathcal{E}_{\text {crit }}$ ) exponentially with time. Using the linear fit, we compare the decay/growth rates with the QNM spectrum predictions, see figure 8:

$$
\left|\frac{\operatorname{Im}(\omega)_{\text {fit }}}{\operatorname{Im}(\omega)_{\mathrm{QNM}}}-1\right| \lesssim\left\{\begin{array}{ll}
10^{-5}, & \mathcal{E}>\mathcal{E}_{\text {crit }} \\
10^{-6}, & \mathcal{E}<\mathcal{E}_{\text {crit }}
\end{array} .\right.
$$

\subsubsection{Unstable sBBL dynamics}

Having reproduced the phase diagram and the linearized symmetry breaking dynamics of sBBL model in section 3.2.1, we now present fully nonlinear dynamical results. We focus on the unstable case only, as for simulations with $\mathcal{E}>\mathcal{E}_{\text {crit }}$, after a brief non-linear regime, the system evolves to symmetric equilibrium configurations discussed above.

We consider first the evolution with $g=-8$ and $\mathcal{E}=0.42978(1) \mathcal{E}_{\text {crit }}$ and $\mathcal{E}_{\text {crit }}$ as in (3.15). Recall that for this value of the coupling $\mathcal{E}_{\text {crit }}>0$. Figures 13-14 collect results for the evolution of the expectation values of operators $\mathcal{O}_{i}, \mathcal{O}_{r}$, the entropy density $s$ and the Kretschmann scalar $K^{h}$ evaluated at the apparent horizon,

$$
K^{h}=\left.R_{a b c d} R^{a b c d}\right|_{(t, r=1)},
$$

relative to the $A d S_{4}$ Kretschmann scalar $K_{A d S_{4}}$ (recall $K_{A d S_{4}}=$ const $=24$ ). Different color coding on the plots represents numerical runs from the same initial conditions, but 

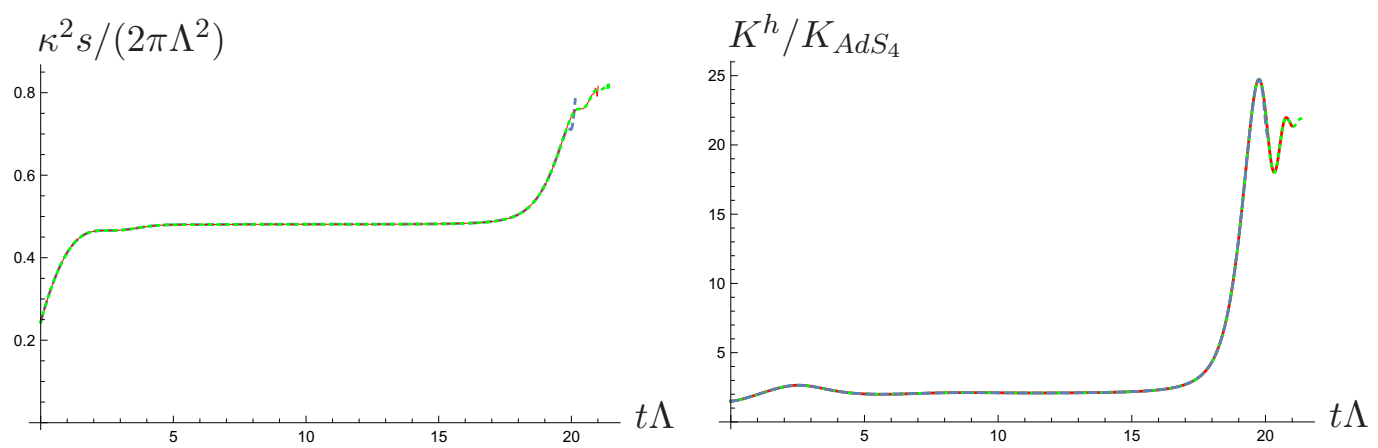

Figure 14. Evolution of the entropy density $s$ and the Kretschmann scalar $K^{h}$ evaluated at the apparent horizon in dynamics of sBBL model with $g=-8$ in the unstable regime, i.e., with $\mathcal{E}<\mathcal{E}_{\text {crit }}$. Different color coding represents different spatial resolutions of the numerical runs.
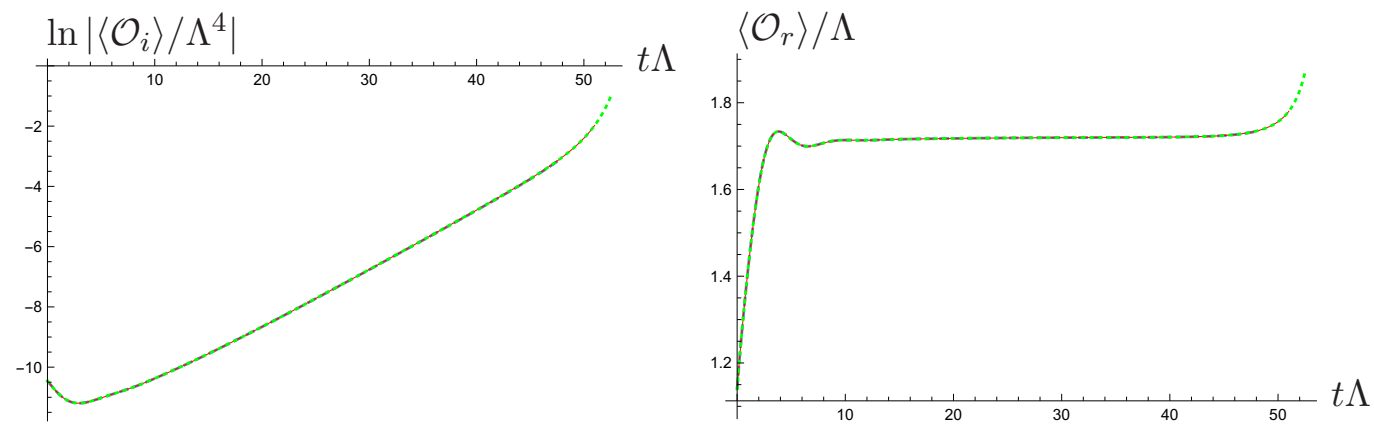

Figure 15. Evolution of expectation values of $\mathcal{O}_{i}$ and $\mathcal{O}_{r}$ operators in sBBL model with $g=-2$ in the unstable regime, i.e., with $\mathcal{E}<\mathcal{E}_{\text {crit }}$. Different color coding represents different spatial resolutions of the numerical runs.

with different spatial relation — the different number of collocation points:

$$
N_{\text {collocation }}=\left\{\begin{array}{ll}
20, & \text { blue dashed } \\
40, & \text { red } \\
60, & \text { green dotted }
\end{array} .\right.
$$

As for the BBL model in [1], the numerical code always crashes, albeit at a slightly later time with increasing spatial resolution. Similar to BBL model, instability in sBBL model persists in a nonlinear regime - we do not see the saturation in expectation values of $\mathcal{O}_{i}$ and $\mathcal{O}_{r}$. However, contrary to BBL, in sBBL model with $g=-8\left(\mathcal{E}_{\text {crit }}>0\right)$ we do not see a clear signature of the divergence of the area density of the apparent horizon (the entropy density); moreover, there is no obvious divergence in the Kretschmann scalar $K^{h}$ as well. Clearly, a better (different) implementation ${ }^{12}$ of the code is needed to answer conclusively whether the entropy density and/or the Kretschmann scalar remain finite.

Figures 15-16 collect the same data, but for the sBBL coupling constant $g=-2$ and $\mathcal{E}=1.0454(8) \mathcal{E}_{\text {crit }}$ with the negative critical energy as in (3.15). Here we have a stronger indication for the divergence of the Kretschmann scalar $K^{h}$ along with the more dramatic

\footnotetext{
${ }^{12} \mathrm{~A}$ finite-difference code as in [28] might be more suitable to capture high-gradients in the evolution. Even more leverage could be achieved with adaptive mesh refinement as in [29].
} 

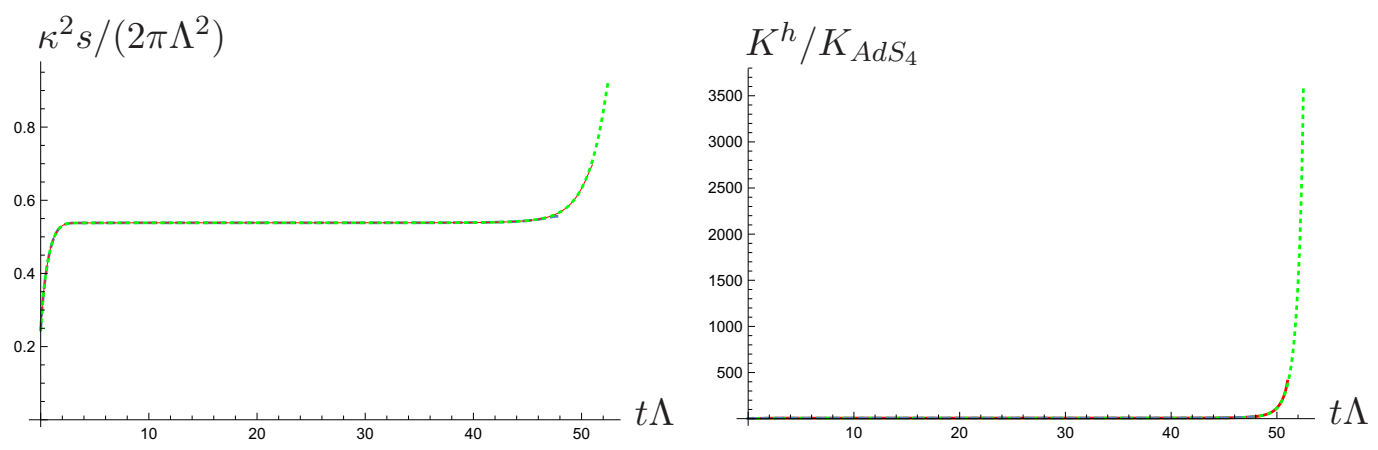

Figure 16. Evolution of the entropy density $s$ and the Kretschmann scalar $K^{h}$ evaluated at the apparent horizon in dynamics of sBBL model with $g=-2$ in the unstable regime, i.e., with $\mathcal{E}<\mathcal{E}_{\text {crit }}$. Different color coding represents different spatial resolutions of the numerical runs.

growth of the entropy density $s$. Better implementation of the numerics could answer whether dynamics in sBBL model at $g=-2$ is physically different from that at $g=-8$, and whether this difference is attributed to the sign of $\mathcal{E}_{\text {crit }}$.

\section{Conclusions}

In [1] it was argued that the phenomenological holographic model introduced in [17] violates the weak cosmic censorship conjecture. The gravitational dual describes dynamics of certain $Q F T_{3}$ with spontaneous symmetry breaking, but without an equilibrium ground state below the instability threshold. ${ }^{13}$ An arbitrary weakly curved initial gravitational configuration was shown to evolve, in a finite boundary time, to a configuration with both the divergent area density of the apparent horizon and the Kretschmann scalar evaluated at the horizon. The divergence of the area density signals that the singularity mechanism is robust - while the evolution was restricted to spatially homogeneous and isotropic states of the $Q F T_{3}$, any finite entropy density state with broken (boundary) spatial translational invariance and/or rotations can not dominate late time dynamics. Following the gravity-fluid correspondence [5], the BBL holographic model points to a development of the singularities from regular initial conditions in corresponding relativistic fluid mechanics.

In this paper we attempted to address two questions:

(1) can BBL scenario be realized in a top-down string construction?

(2) what is the role of supersymmetry on the singularity development?

Concerning (1), it was pointed out in [1] that consistent truncations studied in [21] exhibit the exotic thermodynamics of [17] in grand canonical ensemble. We studied here the corresponding models in details and established that there is no run-away instability in DG models in microcanonical ensemble - rather, we found yet another realization of the mean-field spontaneous symmetry breaking mechanism of $[15,16]$.

Concerning (2), we showed that it is straightforward to modify BBL model to mimic the structure of the bulk scalar coupling to gravity ubiquitous in gauged supergravity consistent

\footnotetext{
${ }^{13}$ Potentially related phenomenon was reported in [30].
} 
truncations $[24,25]$. The scalar potential of the "supersymmetric" generalization of the BBL model (called sBBL here) remains unbounded from below. The equilibrium phase diagram and the linearized symmetry breaking dynamics in BBL and sBBL models are conceptually identical. Further studies (and a better numerical code) are needed to firmly establish whether sBBL model also evolves to a geometry with divergent area density of the apparent horizon and the curvature. As we pointed out, the latter might depend on details of the scalar superpotential, i.e., the nonlinear coupling $g$.

Finally, the challenge remains to find embedding of BBL mechanism in string theory.

\section{A Numerical setup}

We adapt numerical code developed in [1] to study dynamics of spatially homogeneous and isotropic states in DG and sBBL models.

\section{A.1 DG-B model}

We introduce a new radial coordinate

$$
x \equiv \frac{1}{r} \in[0,1], \quad d_{+}=\partial_{t}+A(t, r) \partial_{r} \rightarrow \partial_{t}-x^{2} A(t, x) \partial_{x},
$$

maintaining ${ }^{\prime} \equiv \partial_{x}$ and ${ }^{\cdot} \equiv \partial_{t}$, and redefine the fields

$$
\left\{\Sigma, A, d_{+} \Sigma\right\} \rightarrow\{\sigma, a, d \sigma\}
$$

as follows

$$
\begin{aligned}
\Sigma(t, x) & =\frac{1}{x}+\sigma(t, x), \\
A & =a(t, x)-\Sigma(t, x)^{2}+\frac{2}{x} \Sigma(t, x)+2 \lambda(t) \Sigma(t, x), \\
d_{+} \Sigma(t, x) & =x d \sigma(t, x)+\Sigma(t, x)^{2}-\frac{1}{x} \Sigma(t, x)+\frac{1+x \lambda(t)}{x^{2}} .
\end{aligned}
$$

Using (2.33) and (2.38), we find the asymptotic boundary expansion $x \rightarrow 0_{+}$for all the fields:

$$
\begin{aligned}
f_{i} & =f_{i, 1}(t) x+\mathcal{O}\left(x^{2}\right), \quad d_{+} f_{i}=-f_{i, 1}(t)+\mathcal{O}\left(x^{2}\right), \\
\sigma & =\lambda(t)-\frac{1}{2}\left(f_{1,1}(t)^{2}+f_{2,1}(t)^{2}\right) x+\mathcal{O}\left(x^{2}\right), \\
d \sigma & =m+\frac{\lambda(t)}{2}\left(f_{1,1}(t)^{2}+f_{2,1}(t)^{2}\right)+\mathcal{O}(x), \\
a & =-\dot{\lambda}(t)-f_{1,1}(t)^{2}-f_{2,1}(t)^{2}+m x+\mathcal{O}\left(x^{2}\right), \\
a_{1} & =q_{1} x+\mathcal{O}\left(x^{2}\right)
\end{aligned}
$$

where we set

$$
\left\{A_{1}(t), a_{1,1}(t)\right\}=\left\{m, q_{1}\right\} .
$$

Note that the boundary conditions (2.38) are enforced with the absence of $\mathcal{O}(x)$ terms in asymptotic expansion of $d_{+} f_{i}$ in (A.4).

The rest of the code implementation is as in [1]. 


\section{A.2 sBBL model}

We use a radial coordinate $x$ as in (A.1) and employ the following field redefinitions:

$$
\left\{\phi, \chi, \Sigma, A, d_{+} \phi, d_{+} \chi, d_{+} \Sigma\right\} \rightarrow\{p, q, \sigma, a, d p, d q, d \sigma\}
$$

with

$$
\begin{aligned}
\phi(t, x) & =p(t, x) \\
\chi(t, x) & =x^{3} q(t, x) \\
\Sigma(t, x) & =\frac{1}{x}+\sigma(t, x) \\
A & =a(t, x)+\frac{1}{2} \Sigma(t, x)^{2}-\frac{1}{x} \Sigma(t, x)+\frac{1+x \lambda(t)}{x^{2}}, \\
d_{+} \phi(t, x) & =d p(t, x) \\
d_{+} \chi(t, x) & =x^{3} d q(t, x) \\
d_{+} \Sigma(t, x) & =x d \sigma(t, x)+\frac{1}{2} \Sigma(t, x)^{2}-\frac{1}{2 x} \Sigma(t, x)+\frac{1+x \lambda(t)}{2 x^{2}} .
\end{aligned}
$$

Using (3.17), we find the asymptotic boundary expansion $x \rightarrow 0_{+}$for the new fields:

$$
\begin{array}{rlrl}
p & =p_{1}(t) x+\mathcal{O}\left(x^{2}\right), & q & =q_{4}(t) x+\mathcal{O}\left(x^{2}\right), \\
d p & =-\frac{p_{1}(t)}{2}-p_{2} x+\mathcal{O}\left(x^{2}\right), & d q & =-2 q_{4}(t)+\mathcal{O}(x), \\
\sigma & =\lambda(t)-\frac{p_{1}(t)^{2}}{8} x+\mathcal{O}\left(x^{2}\right), & d \sigma & =\frac{\lambda(t) p_{1}(t)^{2}}{16}+\frac{p_{1}(t) p_{2}}{4}+\mu+\mathcal{O}(x), \\
a & =-\frac{p_{1}(t)^{2}}{8}-\dot{\lambda}(t)+\left(\mu+\frac{1}{8} \lambda(t) p_{1}(t)^{2}\right) & x+\mathcal{O}\left(x^{2}\right) .
\end{array}
$$

The rest of the code implementation is as in [1].

\section{Acknowledgments}

Research at Perimeter Institute is supported by the Government of Canada through Industry Canada and by the Province of Ontario through the Ministry of Research \& Innovation. This work was further supported by NSERC through the Discovery Grants program.

Open Access. This article is distributed under the terms of the Creative Commons Attribution License (CC-BY 4.0), which permits any use, distribution and reproduction in any medium, provided the original author(s) and source are credited.

\section{References}

[1] P. Bosch, A. Buchel and L. Lehner, Unstable horizons and singularity development in holography, JHEP 07 (2017) 135 [arXiv:1704.05454] [INSPIRE]. 
[2] J.M. Maldacena, The large $N$ limit of superconformal field theories and supergravity, Adv. Theor. Math. Phys. 2 (1998) 231 [Int. J. Theor. Phys. 38 (1999) 1113] [hep-th/9711200].

[3] O. Aharony, S.S. Gubser, J.M. Maldacena, H. Ooguri and Y. Oz, Large-N field theories, string theory and gravity, Phys. Rept. 323 (2000) 183 [hep-th/9905111] [INSPIRE].

[4] E. Witten, Anti-de Sitter space, thermal phase transition and confinement in gauge theories, Adv. Theor. Math. Phys. 2 (1998) 505 [hep-th/9803131] [inSPIRE].

[5] S. Bhattacharyya, V.E. Hubeny, S. Minwalla and M. Rangamani, Nonlinear fluid dynamics from gravity, JHEP 02 (2008) 045 [arXiv:0712.2456] [inSPIRE].

[6] M.P. Heller, R.A. Janik and P. Witaszczyk, Hydrodynamic gradient expansion in gauge theory plasmas, Phys. Rev. Lett. 110 (2013) 211602 [arXiv: 1302.0697] [INSPIRE].

[7] A. Buchel, M.P. Heller and J. Noronha, Entropy production, hydrodynamics and resurgence in the primordial quark-gluon plasma from holography, Phys. Rev. D 94 (2016) 106011 [arXiv: 1603.05344] [INSPIRE].

[8] E. Berti, V. Cardoso and A.O. Starinets, Quasinormal modes of black holes and black branes, Class. Quant. Grav. 26 (2009) 163001 [arXiv:0905.2975] [INSPIRE].

[9] A. Buchel, M.P. Heller and R.C. Myers, Equilibration rates in a strongly coupled nonconformal quark-gluon plasma, Phys. Rev. Lett. 114 (2015) 251601 [arXiv:1503.07114] [INSPIRE].

[10] J.F. Fuini and L.G. Yaffe, Far-from-equilibrium dynamics of a strongly coupled non-Abelian plasma with non-zero charge density or external magnetic field, JHEP 07 (2015) 116 [arXiv: 1503.07148] [INSPIRE].

[11] R.A. Janik, G. Plewa, H. Soltanpanahi and M. Spalinski, Linearized nonequilibrium dynamics in nonconformal plasma, Phys. Rev. D 91 (2015) 126013 [arXiv: 1503.07149] [INSPIRE].

[12] A. Buchel and A. Day, Universal relaxation in quark-gluon plasma at strong coupling, Phys. Rev. D 92 (2015) 026009 [arXiv: 1505.05012] [INSPIRE].

[13] A. Buchel, A holographic perspective on Gubser-Mitra conjecture, Nucl. Phys. B 731 (2005) 109 [hep-th/0507275] [INSPIRE].

[14] M. Attems et al., Phase transitions, inhomogeneous horizons and second-order hydrodynamics, JHEP 06 (2017) 129 [arXiv: 1703. 02948] [INSPIRE].

[15] S.A. Hartnoll, C.P. Herzog and G.T. Horowitz, Building a holographic superconductor, Phys. Rev. Lett. 101 (2008) 031601 [arXiv:0803.3295] [INSPIRE].

[16] S.A. Hartnoll, C.P. Herzog and G.T. Horowitz, Holographic superconductors, JHEP 12 (2008) 015 [arXiv:0810.1563] [INSPIRE].

[17] A. Buchel and C. Pagnutti, Exotic hairy black holes, Nucl. Phys. B 824 (2010) 85 [arXiv: 0904.1716] [INSPIRE].

[18] A. Buchel and C. Pagnutti, Correlated stability conjecture revisited, Phys. Lett. B 697 (2011) 168 [arXiv:1010.5748] [INSPIRE].

[19] I. Booth, Black hole boundaries, Can. J. Phys. 83 (2005) 1073 [gr-qc/0508107] [InSPIRE].

[20] P. Figueras, V.E. Hubeny, M. Rangamani and S.F. Ross, Dynamical black holes and expanding plasmas, JHEP 04 (2009) 137 [arXiv:0902.4696] [INSPIRE]. 
[21] A. Donos and J.P. Gauntlett, Superfluid black branes in $A d S_{4} \times S^{7}$, JHEP 06 (2011) 053 [arXiv: 1104.4478] [INSPIRE].

[22] O. Aharony, A. Buchel and P. Kerner, The black hole in the throat: thermodynamics of strongly coupled cascading gauge theories, Phys. Rev. D 76 (2007) 086005 [arXiv:0706.1768] [INSPIRE].

[23] P. Bosch, S.R. Green and L. Lehner, Nonlinear evolution and final fate of charged Anti-de Sitter black hole superradiant instability, Phys. Rev. Lett. 116 (2016) 141102 [arXiv: 1601.01384] [INSPIRE].

[24] C.-h. Ahn and K. Woo, Supersymmetric domain wall and $R G$ flow from 4-dimensional gauged $N=8$ supergravity, Nucl. Phys. B 599 (2001) 83 [hep-th/0011121] [INSPIRE].

[25] N. Bobev, N. Halmagyi, K. Pilch and N.P. Warner, Holographic, $N=1$ supersymmetric $R G$ flows on M2 branes, JHEP 09 (2009) 043 [arXiv:0901.2736] [INSPIRE].

[26] A. Núñez and A.O. Starinets, AdS/CFT correspondence, quasinormal modes and thermal correlators in $N=4$ SYM, Phys. Rev. D 67 (2003) 124013 [hep-th/0302026] [INSPIRE].

[27] A. Buchel and A. Karapetyan, De Sitter vacua of strongly interacting QFT, JHEP 03 (2017) 114 [arXiv:1702.01320] [INSPIRE].

[28] A. Buchel, L. Lehner and R.C. Myers, Thermal quenches in $N=2^{*}$ plasmas, JHEP 08 (2012) 049 [arXiv: 1206.6785] [INSPIRE].

[29] A. Buchel, L. Lehner and S.L. Liebling, Scalar collapse in AdS, Phys. Rev. D 86 (2012) 123011 [arXiv:1210.0890] [INSPIRE].

[30] U. Gürsoy, A. Jansen and W. van der Schee, New dynamical instability in asymptotically Anti-de Sitter spacetime, Phys. Rev. D 94 (2016) 061901 [arXiv: 1603.07724] [INSPIRE]. 